\title{
A Modular Multilevel Converter with Ripple-Power Decoupling Channels for Three-Phase MV Adjustable-Speed Drives
}

\author{
Mohamed S. Diab, Student Member, IEEE, Ahmed M. Massoud, Senior Member, IEEE, Shehab Ahmed, \\ Senior Member, IEEE, and Barry W. Williams
}

\begin{abstract}
This paper presents a drive system based on a modular multilevel converter (MMC) with high-frequency magnetic channels between adjacent-arm submodules (SMs), suitable for medium-voltage, high-power three-phase variablespeed machines. The configuration employs chains of dual halfbridge (DHB) modules linking adjacent SMs of three-phase symmetrical arms. The DHB modules are operating as power channels enabling energy exchange to restore the power imbalance among the SM capacitors. This allows arms' ripplepowers to be entirely decoupled through bidirectional power transfer between adjacent-arm SMs, resulting in a near ripplefree SM capacitor voltage profile. Therefore, the MMC common problem of wide voltage fluctuation across SM capacitors is comprehensively solved, independent of the operating frequency. Additionally, a significant reduction in the sizing requirement of SM capacitance is achieved. The configuration is able to drive multi-megawatt machines from standstill to the rated speed at the rated torque operating condition. The operating principle of the proposed MMC configuration is explained and necessary mathematical analysis is derived. Features and viability of the proposed drive system are verified through simulation and experimentation.
\end{abstract}

Index Terms-Dual half-bridge (DHB), high-frequency transformer, low motor speed, medium-voltage (MV) variablespeed drives, modular multilevel converter (MMC), power decoupling, submodule (SM) capacitor voltage-ripple.

\section{INTRODUCTION}

$\mathbf{T}$ HE modular multilevel converter (MMC) has shown promising potential in medium- to high-voltage, highpower applications due to its unique features [1]. In addition to the advantages of state-of-the-art multilevel converter topologies, such as diode-clamped [2], flying-capacitor [3], and cascaded H-bridge converters [4], the MMC offers outstanding features being a highly modular topology that can be easily scaled to high power ratings, with an enhanced

Manuscript received December 24, 2017; revised April 21, 2018; accepted July 13, 2018.

M. S. Diab is with the Department of Electronic and Electrical Engineering, University of Strathclyde, Glasgow G1 1XQ, U.K., and also with the Electrical Engineering Department, Faculty of Engineering, Alexandria University, Alexandria 21544, Egypt (e-mail: mohamed.diab@strath.ac.uk).

A. M. Massoud is with the Department of Electrical Engineering, Qatar University, Doha 2713, Qatar (email: ahmed.massoud@qu.edu.qa).

S. Ahmed is with the Department of Electrical and Computer Engineering, Texas A\&M University at Qatar, Doha 23874, Qatar (email: shehab.ahmed@qatar.tamu.edu).

B. W. Williams is with the Department of Electronic and Electrical Engineering, University of Strathclyde, Glasgow G1 1XQ, U.K. (e-mail: barry.williams@strath.ac.uk). reliability [5]. The MMC is a popular topology in constantvoltage constant-frequency applications, where it is considered a standard commercialized converter interface in high-voltage direct-current transmission systems. However, utilization of MMC in applications with variable-frequency scenarios, such as adjustable-speed drives, is less common due to the serious challenge associated with its operating principals. With fundamental and second-order ripple powers pulsating simultaneously in MMC arms, the submodule (SM) floating capacitors experience wide voltage fluctuations, particularly at low operating frequencies, that could threaten the safety of switching devices and adversely affect the MMC normal operation. To maintain SM capacitor voltage-ripple within tolerances, the SM capacitance should be designed fairly large to compensate for the high voltage-ripple, which increases the converter volume and weight, while further increases the system stored energy. Nonetheless, at near zero frequency, the SM capacitor voltage undergoes a unidirectional change with extreme voltage-ripple, where increasing the SM capacitance is not viable. This inherent constraint dispossesses the MMC from many medium-voltage (MV) drives applications, where operation from standstill at rated current and continuous lowspeed operation are required. This restricts its utilization to a range of quadratic-torque loads such as pumps and compressors [6], [7].

Several approaches have been proposed in diversified studies addressing SM capacitor voltage-ripple in variablespeed drives applications. One effective approach is to inject a high-frequency (HF) circulating current into the MMC phase arms, while injecting the same frequency harmonic into the converter output common-mode (CM) voltage [6]-[11]. That is, the low-frequency pulsating powers in the MMC arms are transformed into HF components, allowing the SM capacitors to be charged and discharged more frequently such that their voltage ripple is attenuated. Although this compensation approach limits capacitor voltage variation at low motor speeds, the CM voltage introduced at the motor terminals has negative impact on both motor insulation and bearings, shortening its lifetime. Also, the high magnitude of the HF arm currents significantly increases current stress, and consequently power loss, which necessitates overrating the current capacity of the switching devices.

Other solutions investigated SM capacitor voltage-ripple suppression from different perspectives. The control methods in [12] and [13], and the operation modes in [14] and [15] were adopted to improve the SM capacitor voltage-ripple 
profile, sacrificing some MMC principal features. Various modifications to the basic MMC structure have been discussed in an attempt to find a hardware solution to the power imbalance problem between MMC arms. In this context, physical power transfer channels between the upper and lower MMC arms were proposed in [16] and [17] to redistribute the power between arms. In addition, different SM structures, rather than the conventional half-bridge SM (HB-SM), were employed in [18] and [19].

Although most of the mentioned approaches satisfactorily suppress SM capacitor voltage-ripple at low operating frequencies, they are unable to drive a machine at full-load torque from standstill condition. Also, no detailed verification or results have been demonstrated for continuous lowfrequency operation of high-power machines in multimegawatt drive systems.

The authors recently proposed a ripple-power decoupling approach for MV high-power MMC-fed variable-speed drives incorporating open-end stator winding machines [20]. This approach overcomes the inherent limitations of MMC-fed drives by counter-balancing the fundamental ripple-power of each two adjacent arms, with a common machine winding, by employing modular energy-exchange schemes between adjacent-arm SMs operated with out-of-phase modulation. That is, the excess of the fundamental capacitive energy stored at one arm side is transferred to the adjacent oppositely modulated arm, to cover for the lack of energy in that arm. This results in a significant reduction in SM capacitor voltage ripple, where the fundamental ripple component is entirely eliminated, rendering the SM capacitor voltage pulsating due to only the second-order ripple component. Similar research has been presented in [21] and [22], but with the fundamental ripple power counter-balanced between each pair of upperand lower-arm SMs of the same MMC phase-leg.

In this paper, the authors extend the ripple-power decoupling approach to compensate for both the fundamental and secondorder ripple power components in the MMC arms. That is, a new MMC drive system configuration is proposed for variable-speed drives employing three-phase machines with star/delta connections, with modular energy-exchange chainlinks interfacing each three adjacent-arm SMs through isolated dc-dc converters. With symmetrical modulation of the three MMC phase-legs, the pulsating powers (manifested as capacitor voltage ripple) in each three adjacent-arm SMs can be eliminated altogether, since their vectorial sum is zero. This occurs by transferring the ripple powers between three magnetically-interfaced SMs, of different three phase-legs, in accordance to a capacitor voltage-ripple control scheme that ensures ripple free SM capacitor voltage. The decoupling of the ripple powers of MMC arms results in a significant reduction in the SM capacitance and MMC stored energy. Further, it enables the MMC to drive a variable-speed machine at constant torque over the entire speed range, even at a standstill. It is worth mentioning that the added energy balancing hardware retains the MMC modularity feature, and its control is independent of the main MMC system control loops.

This paper is presented as follows. Section II illustrates the problem to be solved by presenting the analysis of MMC arm

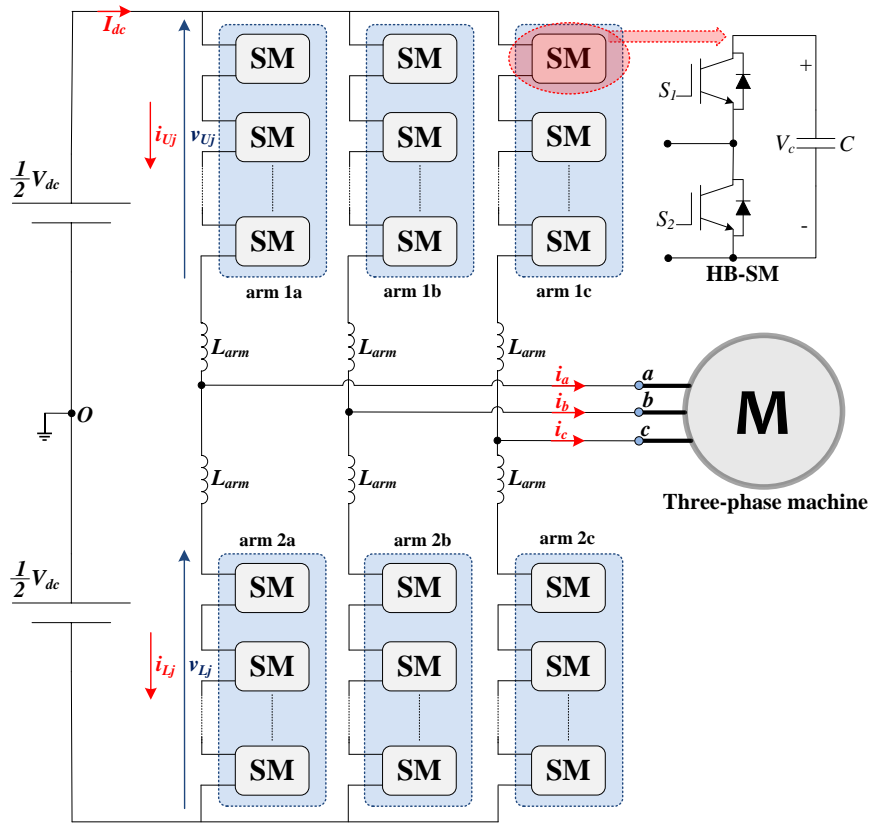

Fig. 1 Circuit diagram of a conventional MMC topology feeding a three-phase induction machine.

power and SM capacitor voltage-ripple to identify their different frequency components. The proposed MMC configuration is illustrated in Section III. The drive system control algorithms are discussed in Section IV. Section V briefly discusses the proposed configuration and its possible variations. Simulation results for multi-megawatt MMC systems and experimental results for a downscaled laboratory prototype are presented in section VI. Finally, Section VII concludes the contributions of this paper.

\section{RIPPLE ANALYSIS AND CHARACTERIZATION OF CONVENTIONAL MMC}

The circuit diagram of a three-phase MMC feeding an induction machine is shown in Fig. 1. Each MMC phase-leg is formed by two series arms connected through arm inductors $L_{\text {arm }}$, while each arm consists of $N$ series-connected SMs. The SM commonly consists of an HB cell with a dc capacitor of an equivalent capacitance $C$ and a rated voltage $V_{c}$.

The output voltage of an MMC phase-leg is denoted by $v_{j o}$, while the current through a machine winding is denoted by $i_{j}$, where $j \in\{a, b, c\}$, and are expressed as follows.

$$
\left\{\begin{array}{c}
v_{j o}=V_{o} \cos \left(\omega t+\theta_{j}\right) \\
i_{j}=I_{o} \cos \left(\omega t+\theta_{j}-\varphi\right)
\end{array}\right.
$$

where $V_{o}$ and $I_{o}$ are the voltage and current amplitudes, respectively, $\omega$ is the output angular frequency, $\theta_{j}$ is the phase angle of the stator voltage $\left(\theta_{a}=0^{\circ}, \theta_{b}=120^{\circ}\right.$, and $\left.\theta_{c}=240^{\circ}\right)$, and $\varphi$ is the machine power-factor angle.

The magnitude of the ac output voltage is defined by the modulation index $M$ and the voltage of the input source $V_{d c}$, as follows.

$$
V_{o}=\frac{1}{2} M V_{d c}
$$


The MMC arm voltages in addition to the arm currents are given by (3) and (4), respectively, with the subscripts $U$ and $L$ referring to the corresponding 'upper' and 'lower' arm.

$$
\begin{array}{r}
\left\{\begin{array}{l}
v_{U j}=\frac{1}{2} V_{d c}-v_{j o} \\
v_{L j}=\frac{1}{2} V_{d c}+v_{j o}
\end{array}\right. \\
\left\{\begin{array}{l}
i_{U j}=i_{c m j}+\frac{1}{2} i_{j} \\
i_{L j}=i_{c m j}-\frac{1}{2} i_{j}
\end{array}\right.
\end{array}
$$

where $i_{c m j}$ is the CM current of a phase-leg $j$, and referred to as the circulating current. With appropriate even-order harmonic suppression control [23], the CM current can be considered as a dc component which can be calculated through a lossless power balance between the dc input and ac output as shown in (5), where $I_{d c}$ is the MMC dc input current.

$$
i_{c m j}=\frac{I_{d c}}{3}=\frac{M I_{o} \cos \varphi}{4}
$$

The instantaneous power input to each arm is the product of the arm voltage (3) and the corresponding arm current (4):

$$
\left\{\begin{array}{l}
p_{U j}=v_{U j} i_{U j} \\
p_{L j}=v_{L j} i_{L j}
\end{array}\right.
$$

Substituting (1)-(5) into (6) and arranging terms yields,

$$
\left\{\begin{array}{l}
p_{U j}=p_{c m}+p_{d m} \\
p_{L j}=p_{c m}-p_{d m}
\end{array}\right.
$$

where $p_{c m}$ and $p_{d m}$ are the $\mathrm{CM}$ and the differential-mode (DM) components of the arm power, respectively, and are written as follows:

$$
\begin{gathered}
p_{c m}=-\frac{V_{d c} I_{o} M}{8} \cos \left[2\left(\omega t+\theta_{j}\right)-\varphi\right] \\
p_{d m}=\frac{V_{d c} I_{o}}{8} \sqrt{4+\cos ^{2}(\varphi)\left(M^{4}-4 M^{2}\right)} \cos \left(\omega t+\theta_{j}-\gamma\right) \\
\gamma=\varphi+\tan ^{-1} \frac{M^{2} \tan \varphi \cos ^{2} \varphi}{2-M^{2} \cos ^{2} \varphi}
\end{gathered}
$$

Equations (7) and (8) establish that the arm power is characterized by two components. The DM component pulsates with the fundamental frequency and appears in antiphase in the upper and lower arms as a consequence of the load current being split differentially between the arms. The $\mathrm{CM}$ component pulsates at twice the fundamental frequency due to the different frequency alternation of both input and output powers. Both CM and DM power components are graphically demonstrated in Fig. 2 along with the total arm power, where it can be noticed that the DM power is the dominant component.

Due to the dual frequency alternation of the MMC arm powers, capacitor voltage-fluctuation has both $\mathrm{CM}$ and DM voltage-ripple components alternating at twice the fundamental frequency and at the fundamental frequency, respectively, as demonstrated by (10).

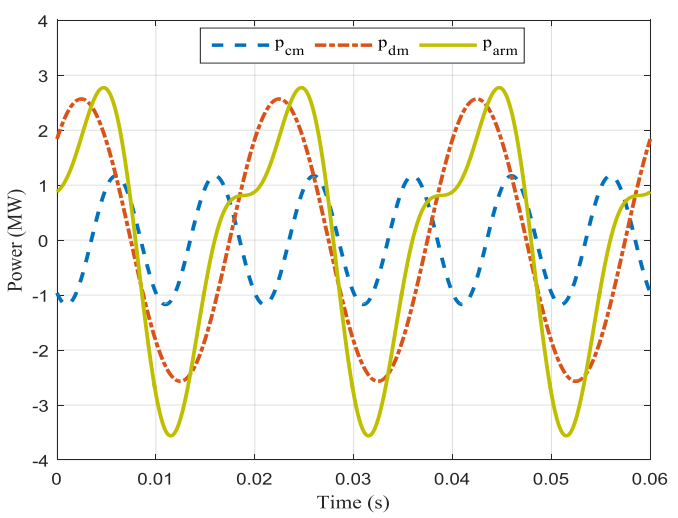

Fig. $2 \mathrm{MMC}$ arm power components. $\left(V_{d c}=25 \mathrm{kV}, I_{o}=500 \mathrm{~A}, M=0.75\right.$, and $\varphi=35^{\circ}$ ).

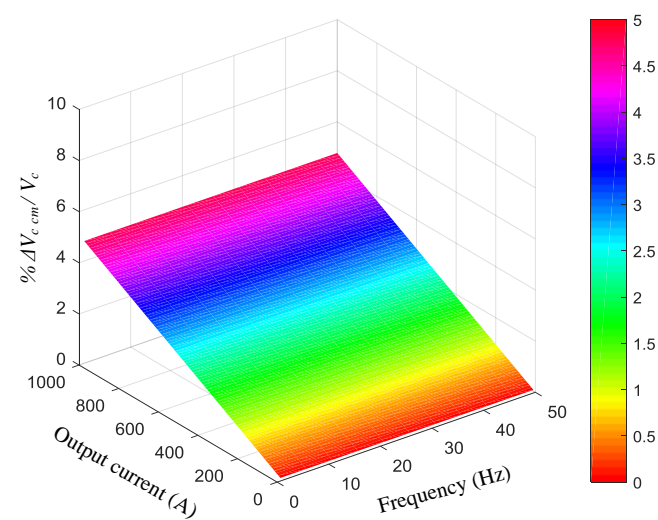

(a)

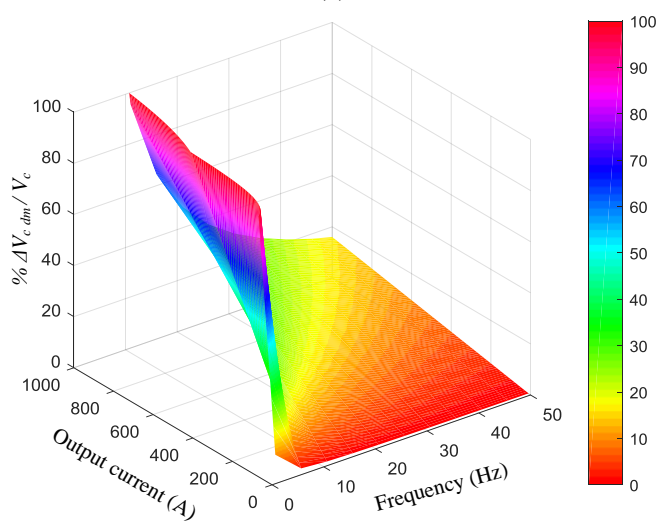

(b)

Fig. 3 Relation curves of capacitor voltage-ripple at constant $M / \omega$ versus operating frequency and output current for (a) CM component and (b) DM component. $\left(C=3 \mathrm{mF}, V_{c}=2.5 \mathrm{kV}\right.$, and $\left.\varphi=35^{\circ}\right)$

$$
\begin{aligned}
\Delta v_{c_{U j}}=- & \frac{\Delta V_{c_{c m}}}{2} \sin \left[2\left(\omega t+\theta_{j}\right)-\varphi\right] \\
& +\frac{\Delta V_{c_{d m}}}{2} \sin \left(\omega t+\theta_{j}-\gamma\right) \\
\Delta v_{c_{L j}}=- & \frac{\Delta V_{c_{c m}}}{2} \sin \left[2\left(\omega t+\theta_{j}\right)-\varphi\right] \\
& -\frac{\Delta V_{c_{d m}}}{2} \sin \left(\omega t+\theta_{j}-\gamma\right)
\end{aligned}
$$

where $\Delta v_{c}$ and $\Delta V_{c}$ are the capacitor voltage-variation and the absolute peak-to-peak variation of SM capacitor voltageripple, respectively. 


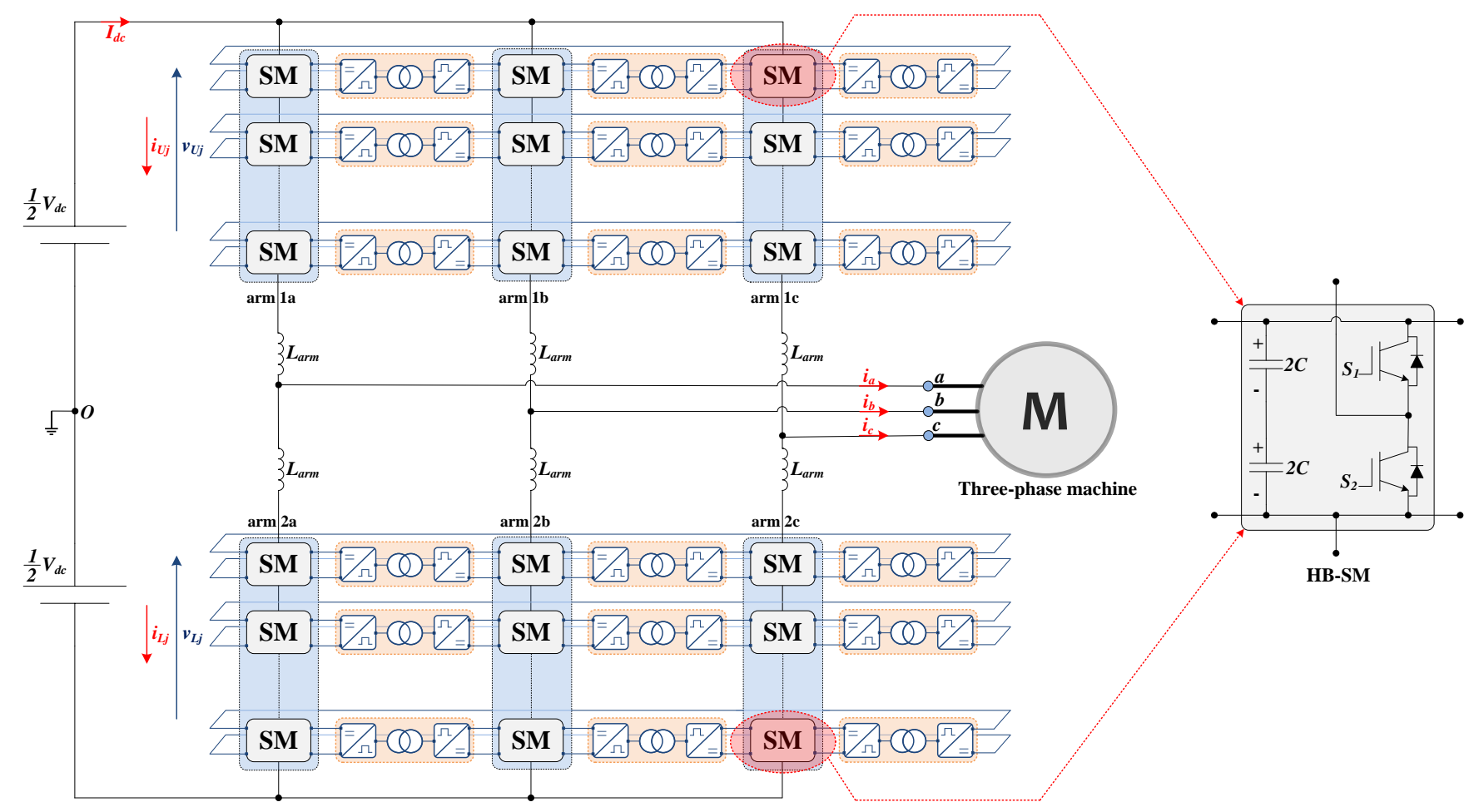

Fig. 4 Circuit diagram for the proposed MMC configuration feeding a three-phase machine.

The magnitudes of the peak-to-peak capacitor voltage-ripple due to CM and DM components are [20], [24]:

$$
\begin{gathered}
\Delta V_{c_{c m}}=\frac{I_{o} M}{8 \omega C} \\
\Delta V_{c_{d m}}=\frac{I_{o}}{4 \omega C} \sqrt{4+\cos ^{2}(\varphi)\left(M^{4}-4 M^{2}\right)}
\end{gathered}
$$

Fig. 3 shows the 3-D curves of the normalized capacitor voltage ripple due to both the CM and DM components, varied with both operating frequency and output current, while the ratio $M / \omega$ is maintained constant as a requirement of constant motor torque condition. Fig. 3a elucidates that the CM component has a slight effect on the capacitor voltage-ripple only with load current increase. That is, for a $1000 \mathrm{~A}$ current increase, the $\mathrm{CM}$ voltage ripple constitutes for $\pm 2.5 \%$, independent of the operating frequency. In contrast, Fig. 3b shows the DM component has a dominant influence in the SM capacitor voltage-ripple, as it is significantly increasing with both operating frequency reduction and output current increase.

\section{PROPosed MMC CONFIGURATION WITH RIPPLE POWER DECOUPLING}

The proposed MMC configuration employs magnetic chains equipped with power-decoupling channels linking adjacentarm SMs. The power-decoupling channels are realized through HF transformer-based $\mathrm{dc}-\mathrm{dc}$ converter units interfacing each adjacent SM of symmetrically modulated phase-arms as shown in Fig. 4, where a three-phase machine is fed through the proposed MMC drive configuration.

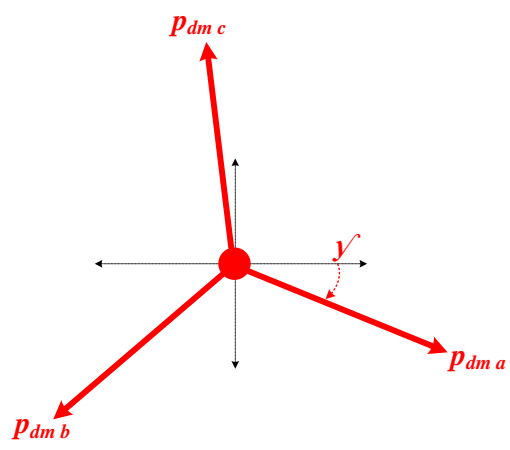

(a)

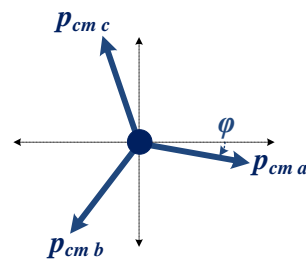

(b)
Fig. 5 Phasor representation for MMC arm power components in the (a) fundamental-frequency domain and (b) second-order frequency domain.

\section{A. Ripple Power Decoupling}

Referring to (8), both the CM and DM power components symmetrically pulsate in the MMC's three-phase arms, as represented in the frequency domain of Fig. 5. The symmetrical alternation of both power components results in a symmetrical fluctuation in the total arm power, as shown in Fig. 6a, which is consequently inherited in the corresponding SM capacitor voltage-variation, as presented in Fig. 6b. That is, the capacitor voltage-ripple of an upper-arm SM alternates with a $2 / 3 \pi$ phase-shift to the capacitor voltage-ripple of adjacent SMs in other upper phase-arms, while it alternates in anti-phase to the capacitor voltage-ripple of an SM in corresponding lower arm due to the predominance of the DM component. 


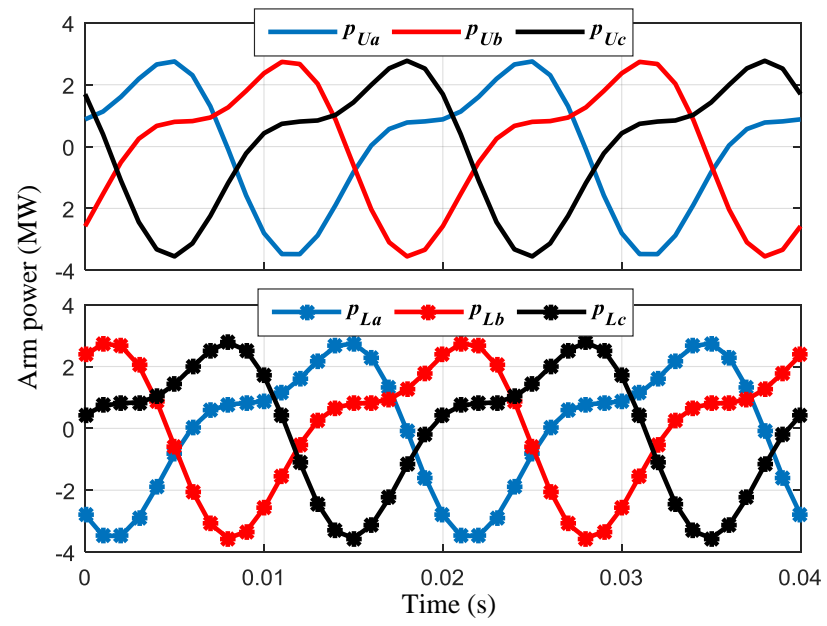

(a)

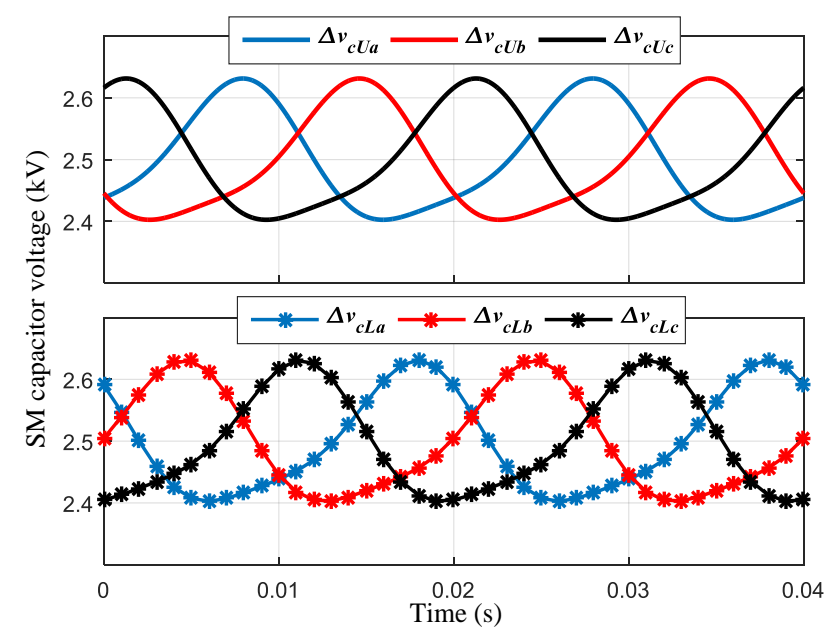

(b)

Fig. 6 Symmetrical alternation of (a) three-phase arm powers and (b) three-phase $\mathrm{SM}$ capacitor voltages. $\left(V_{d c}=25 \mathrm{kV}, V_{c}=2.5 \mathrm{kV}, C=3 \mathrm{mF}, I_{o}=500 \mathrm{~A}, M\right.$ $=0.75$, and $\varphi=35^{\circ}$ ).

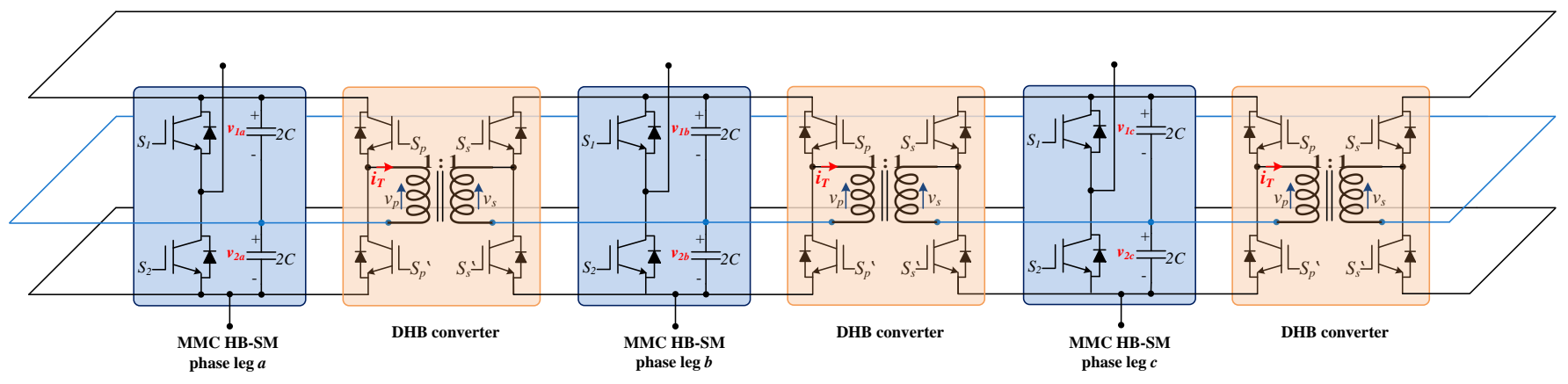

Fig. 7 A DHB-based power decoupling chain for three MMC adjacent-arm HB-SMs.

The basic idea of the active power decoupling is to divert the pulsating power from one specific energy storage component to another, through an active circuit. That is, the proposed MMC configuration redistributes the stored capacitive-energy among each three adjacent-arm SMs by allowing their ripple power to be transferred from one SM with an excess of capacitive stored energy to the other SMs, in a closed magnetic loop, to source the lack of energy there. With zero vectorial sum of symmetrical three-phase components, both the CM and DM power components are decoupled altogether in each chain link of adjacent-arm SMs. This results, ideally, in a ripple-free SM capacitor voltage profile.

\section{B. Isolated Power Decoupling Channels}

The dual active-bridge (DAB) converter is used in a variety of isolated dc-dc power conversion applications [25]. It has the merit of achieving high density bidirectional power transfer with galvanic isolation, soft-switching of semiconductor devices, and modular structure. The half-bridge version of such a converter, denoted as a dual half-bridge (DHB), requires half the number of switching devices as the full-bridge version, offering the benefits of low device count, reduced size, and operation with zero steady-state dc offset of transformer magnetizing current [26], [27]. In this paper, the DHB converter is employed as a dc-dc energy-exchange unit that achieves energy rebalancing for MMC SMs. A detailed circuit diagram for one of the magnetic chains linking adjacent-arm SMs, shown in Fig. 4, is further illustrated in Fig. 7, where three HB-SMs are interconnected together through three DHB converters.

The DHB converter is composed by a pair of voltage-fed HB inverters interfaced through an $\mathrm{HF}$ transformer. The transformer turns ratio is unity since the dc-dc conversion is only employed for energy balancing between bridge sides at the same voltage level. DHB parasitic components are exploited to achieve some operational characteristics, where the leakage inductance of the HF transformer is employed as an energy transfer element between the dc capacitors, while the output capacitance of switching devices is used to realize soft-switching operation.

\section{DHB Principle of Operation}

Since the DHB circuit creates a square-wave voltage on each side of the transformer, the power flow is controlled via the phase angle difference, $\delta$, between the primary and secondary voltage vectors. The most common approach for square-wave generation at both converter side is the phase-shift PWM in which each converter side is modulated with a triangular carrier signal of a frequency $\omega_{h}$ and a constant duty ratio, $D$, of $50 \%(D=0.5)$. Depending on the phase angle difference between the primary and secondary voltages, the amount of power transfer is [20]: 


$$
P=\frac{V_{c}^{2} \delta(\pi-|\delta|)}{8 \pi^{2} f_{h} L}
$$

where $L$ is the transformer leakage inductance and $f_{h}$ is the DHB switching frequency.

To generate a phase difference $\delta$ between both DHB converter sides, two modulation signals are defined as follows [22].

$$
\begin{gathered}
M_{1}=D-\frac{\delta}{\pi} \\
M_{2}=\left|1-M_{1}\right|
\end{gathered}
$$

According to the sign of $\delta$, bidirectional power can be transferred between DHB converter sides as illustrated in Fig. 8 , where $v_{p}$ and $v_{s}$ are the voltages applied on the primary and secondary transformer sides respectively.

\section{DHB Soft Switching}

DAB converters offer natural zero-voltage-switching (ZVS) transitions for the turn-on of all devices. A snubber capacitor should be connected across each switching device to extend the ZVS action to the turn-off instants. The ZVS condition for both switching devices of each $\mathrm{HB}$ is that the net current leaving the leg pole (middle access-point of the leg) lags the voltage of the pole. With the necessity of inserting a dead time between switching devices in the same bridge leg, the net leg output current should not change direction during the dead time until the opposite switching device is turned on. During the dead time, this current charges one snubber capacitor and discharges the other in the same leg, resulting in a soft $d v / d t$. For turn-on ZVS, each switching device at each bridge side should have a negative current just before turning on, such that the anti-parallel diode is conducting, while the voltage across the switching device is almost zero. On the other side, when the switching device is turned off, the voltage across the snubber capacitor is almost zero and the switching device current will divert to the snubber capacitor, which slightly increases the snubber capacitor voltage at the turn-off instant.

Since the proposed MMC configuration employs DHB modules with a unity voltage conversion ratio, ZVS operation is ensured for any loading condition [25].

\section{Operation at Zero Motor-Speed/frequency}

In a conventional MMC topology, the unidirectional current through MMC arms during near zero frequency operation results in a unidirectional change in SM capacitor voltage, leading to extreme capacitor voltage-ripple. In contrast, the proposed MMC-DHB configuration is prominently advantageous being able to efficiently operate at near zero frequency, that is, driving a machine from a full load torque standstill condition, with an enhanced SM capacitor voltage ripple-profile. At near zero frequency, the $\mathrm{CM}$ power in the different MMC arms is almost zero as a consequence of the power at both input and output ports being dc. Also, the DM symmetrical power components pulsating in the MMC arms are counter-balanced within the magnetic power-decoupling chains interfacing each group of adjacent-arm SMs.

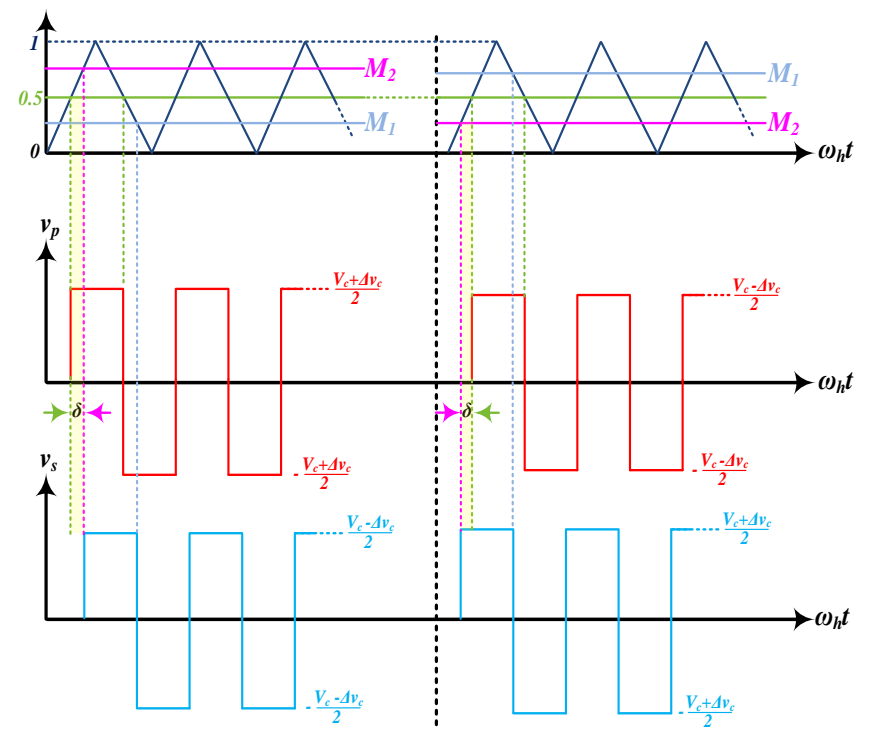

(a)

(b)

Fig. 8 Sketch of the phase-shift PWM of a DHB converter for bidirectional power transfer. (a) Positive phase-shift angle and (b) Negative phase-shift angle.

\section{CONTROL AlgorithMS}

In this section, the control schemes applied in the operation of the proposed MMC-DHB system as a variable-speed motor drive are illustrated. A block diagram for the overall control algorithm is shown in Fig. 9. It is divided into three main parts: a) motor control, b) MMC control, and c) the DHB power-channels control. Each part of the control system is briefly described in the following subsections. The outputs of the overall control algorithm are the switching commands for both MMC SMs and the DHB power channels.

\section{A. Motor Control}

The field-oriented control (FOC) scheme is applied to control the operational characteristics of the induction motor. As shown in the block diagram of Fig. 9, the FOC employs two control loops for both the machine torque and flux, implemented in the $d q$ rotating frame, where two reference values for motor speed and flux are fed to the FOC scheme to generate the commanded values of torque- and flux-producing components of the stator current $\left(i^{*}{ }_{q}\right.$ and $\left.i^{*}{ }_{d}\right)$. The reference current components are processed through two independent pairs of proportional integral (PI) controllers to generate two sets of reference voltage vectors $\left(v^{*}{ }_{q}\right.$ and $\left.v^{*}{ }_{d}\right)$. These vectors are used to generate a set of three-phase reference voltages $\left(v^{*}{ }_{a}, v^{*}{ }_{b}\right.$, and $\left.v^{*}{ }_{c}\right)$, that are utilized in the MMC modulation as will be explained.

\section{B. MMC Control}

Each MMC phase-leg is controlled by a modulator that commands a varying number of SMs to be inserted at each time instant according to a phase disposition PWM scheme. Each modulator operates according to a sinusoidal reference input signal which depends on the motor control requirements. To maintain all of the MMC paralleled phase-legs balanced, a capacitor voltage balancing algorithm in addition to a CM current control scheme are applied as follows. 


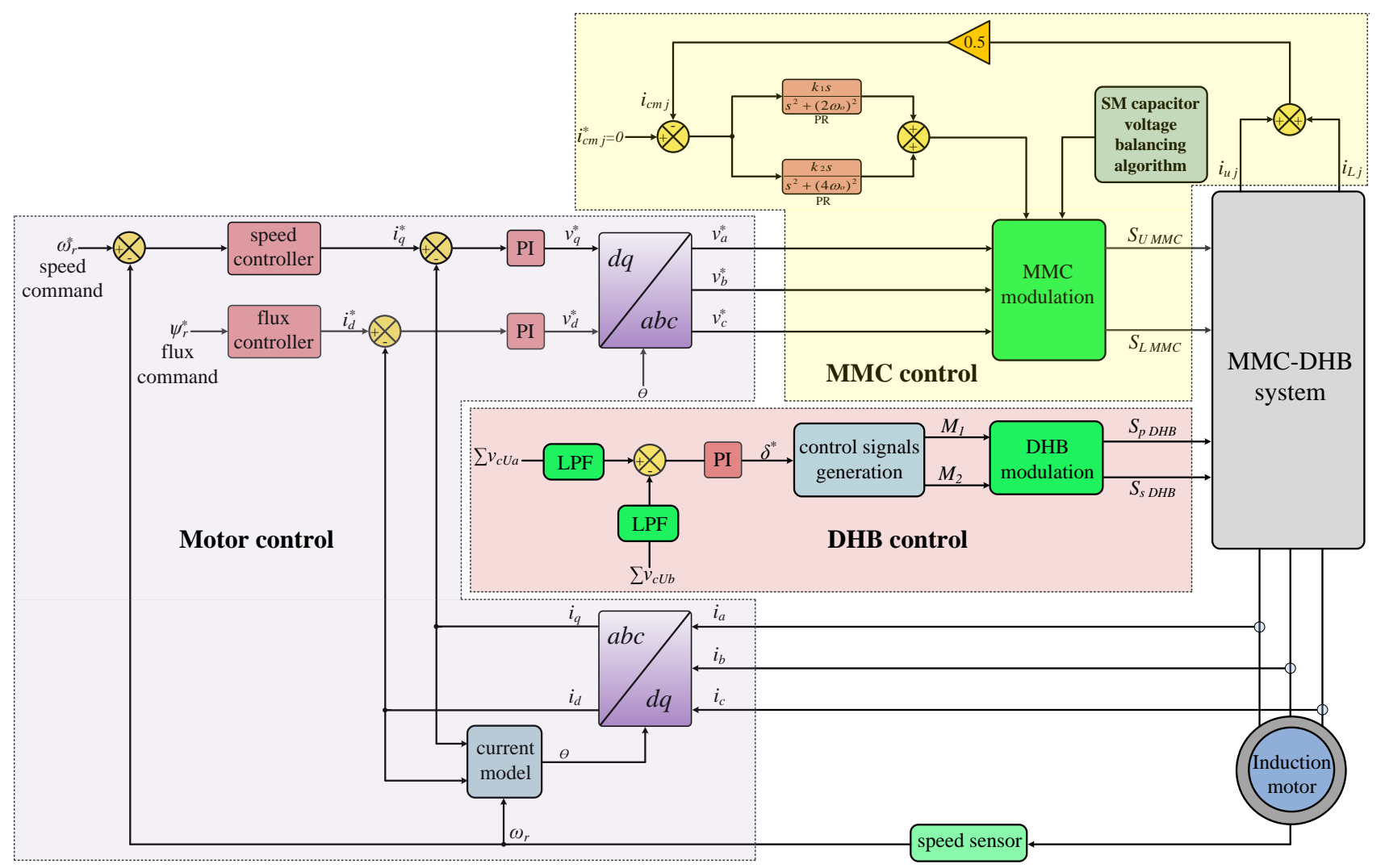

Fig. 9 Control block diagram for the proposed MMC-DHB motor drive system.

\section{Capacitor Voltage Balancing}

SM capacitor voltage balancing is maintained by a selection mechanism based on capacitor voltage measurements at each switching instant. This mechanism sorts the SM capacitor voltages, and then decides which individual SM be inserted or bypassed, according to the arm current direction [28].

\section{CM Current Regulation}

A closed-loop control scheme for even-harmonics suppression of the CM current is implemented as introduced in [23], with the advantage of being independent of the SM capacitor voltage balancing. The controller consists of paralleled resonant controllers with the resonant frequency set at the even harmonics. Since the second- and fourth-order harmonics are the most dominant components in the CM current, only two proportional resonant (PR) controllers are utilized and tuned at $2 \omega_{o}$ and $4 \omega_{o}$, where $\omega_{o}$ is the fundamental angular frequency. The reference $\mathrm{CM}$ current is set to zero, while the outputs of the paralleled PR controllers are added together and fed to the MMC modulator as correction parameters for the three-phase reference signals.

\section{DHB Control}

The control of the DHB modules is ultimately to track both the level and direction of power exchange within each chain of interfaced MMC SMs, which rebalances the stored capacitive energy among the SMs. To ensure each SM capacitor voltage is ripple-free, a control loop employing a PI controller is implemented for each DHB module. The PI controller sets the voltage difference of each DHB primary- and secondary-side capacitor pair to zero, providing the necessary phase angle difference, $\delta^{*}$, which is utilized as an input to the DHB modulator. Accordingly, the DHB modulator generates the control signals $M_{1}$ and $M_{2}$, as given by (13), such that they are compared with a triangular carrier signal to generate the DHB switching signals $\left(S_{p D H B}\right.$ and $\left.S_{S D H B}\right)$, resulting in square wave voltages at both sides of the DHB module, shifted by the commanded angle $\delta^{*}$. With a fast and adequate modulation of the MMC arms, the same phase angle difference is utilized for all the $N$ power channels located at the same arm. That is, in the DHB control blocks of Fig. 9, the sum of the SM capacitor voltages of the upper arm of phase $a\left(\sum v_{c U a}\right)$, are subtracted from that of the upper arm of phase $b\left(\sum v_{c u b}\right)$ to control the power flow through the DHB modules interconnecting the upper SMs of both phase $a$ and $b$. The low-pass filter (LPF) is utilized to remove any harmonic wave distortion. The same control loop is repeated for each set of DHBs interfacing the SMs of two adjacent arms, where six PI controllers are employed to control all DHB modules.

\section{DISCUSSION}

The proposed approach in this paper employs power decoupling chain-links based on DHB units acting as SM energy balancers. In the MMC-DHB configuration shown in Fig. 4 (denoted as configuration 1), each magnetic chain link interfaces three MMC SMs through three DHB units. Therefore, the total number of employed DHB units is equal to the total number of MMC SMs.

\section{A. DHB Rating}

Although the proposed approach entirely decouples the SM ripple power (both CM and DM components), the decoupled 
power can be approximated as the DM power, being the major contributor in the arm ripple power. That is, the maximum SM decoupled power can be calculated from (8b), as:

$$
P_{S M_{\max }}=\frac{P_{D M_{\max }}}{N}=\frac{V_{d c} I_{o}}{4 N}
$$

where $P_{S M_{\max }}$ and $P_{D M_{\max }}$ are the peak values of the decoupled SM power and the DM arm power, respectively. Since each SM capacitor is shared between two DHB units, the SM ripple power can be transferred through two power paths. The DHB control algorithm limits the maximum power transferred through each DHB unit to be equal to half the SM decoupled power. That is, each DHB unit is rated at half the SM power as given by (15), where $P_{D H B_{\max }}$ is the peak value of the power transferred through each DHB unit.

$$
P_{D H B_{\max }}=\frac{P_{S M_{\max }}}{2}=\frac{V_{d c} I_{o}}{8 N}
$$

Since the voltage applied on both transformer windings is $1 / 2 V_{c}$, the maximum transformer current, $I_{T_{\max }}$, is calculated as:

$$
I_{T_{\text {max }}}=\frac{2 P_{D H B_{\max }}}{V_{c}}=\frac{I_{o}}{4}
$$

Therefore, the voltage rating of the DHB switching devices is $V_{c}$, while the current rating is $1 / 4 I_{o}$.

\section{B. MMC-DHB Configuration with Reduced DHB Units}

A variation of the MMC-DHB configuration can be realized as shown in Fig. 10, (denoted as configuration 2), where the number of the employed DHB units is reduced. In this alternative configuration, each three adjacent-arm SMs are linked through a magnetic chain equipped with two DHB units. Referring to Fig. 10, the SMs of the middle phase-leg are explicitly interfaced with the SMs of both the right and left phase-legs through two DHB modules. This keeps SMs at the right and left phase-legs implicitly connected through the corresponding middle phase-leg's SMs. Therefore, the number of employed DHB units in this alternative configuration is reduced to $2 / 3$ the total number of the MMC SMs. However, each DHB module should be rated at the full SM decoupled power, which doubles the current stress of the DHB switching devices, compared to configuration 1. Table I gives a quantitative assessment for the two proposed MMC-DHB configurations, in addition to that presented in [21].

It is worth mentioning that both configurations give the same power-decoupling performance which results in same SM capacitor voltage-ripple profile. However, configuration 1 is preferred with high power drives, since it implies lower power through the DHB modules. Configuration 2 is recommended at lower power ratings, where the volume of the drive system is highly prioritized. With a $166 \mathrm{~kW}$ indicating the maximum power rating of practical implementation of $\mathrm{HF}$ transformers [29], MMC-DHB configuration 1 can be utilized to drive $20 \mathrm{MW}$ machine, with $10 \mathrm{MMC}$ SMs per arm. These parameters are selected for the simulation case study models, as in Section VI.

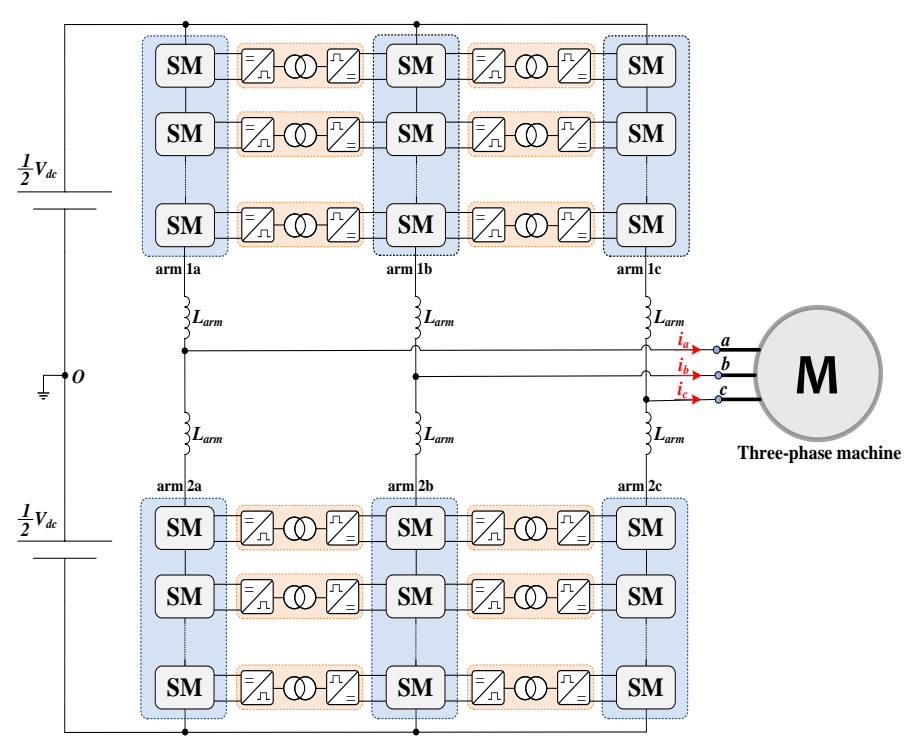

Fig. 10 An alternative structure of the proposed MMC configuration with reduced number of DHB modules.

TABLE I

COMPARISON BETWEEN DIFFERENT MMC-DHB CONFIGURATIONS

\begin{tabular}{lccc}
\hline \hline & Config. 1 & Config. 2 & Config. in [21] \\
\hline \hline Number of DHB modules & $6 \mathrm{~N}$ & $4 \mathrm{~N}$ & $3 \mathrm{~N}$ \\
\hline \hline DHB rated power & $1 / 2 P_{S M}$ & $P_{S M}$ & $P_{S M}$ \\
\hline \hline Semiconductors rated voltage & $1 / 2 V_{c}$ & $1 / 2 V_{c}$ & $1 / 2 V_{c}$ \\
\hline \hline Semiconductors rated current & $1 / 4 I_{O}$ & $1 / 2 I_{O}$ & $1 / 2 I_{o}$ \\
\hline \hline Decoupled ripple component & $\begin{array}{c}\mathrm{CM} \text { and } \\
\mathrm{DM}\end{array}$ & $\begin{array}{c}\mathrm{CM} \text { and } \\
\mathrm{DM}\end{array}$ & $\mathrm{DM}$ \\
\hline \hline
\end{tabular}

\section{VERIFICATION}

The performance of the proposed MMC topology with the DHB power decoupling scheme was investigated using MATLAB/SIMULINK simulation models, in addition to experimentation using a downscaled laboratory prototype. In both verification methods, steady-state and dynamic performances are examined with different operating scenarios, with the parameters listed in Table II.

\section{A. Simulation Verification}

In the simulation study, the MMC-DHB configuration 1 is implemented, where a chain link of three DHB modules interfaces each adjacent-arm SM. The steady-state fundamental waveforms for the MMC system at rated parameters are shown in Fig. 11, while Fig. 12 shows the waveforms of the DHB balancing scheme, where the MMCDHB system is supplying a three-phase $R L$ load. In Fig. 11, both three-phase line voltages and load currents show highquality sinusoidal waveforms. The arm currents have been controlled to be free of both second- and fourth-order harmonics, as explained in Section IV, where the PR controllers are tuned at $100 \mathrm{~Hz}$ and $200 \mathrm{~Hz}$, respectively, resulting in a near dc $\mathrm{CM}$ current. 
TABLE II

SIMULATION AND EXPERIMENTATION PARAMETERS

\begin{tabular}{|c|c|c|}
\hline \multicolumn{3}{|c|}{ MMC parameters } \\
\hline & Simulation & Experiment \\
\hline Number of SMs per arm $(N)$ & 10 & 3 \\
\hline Rated active power & $20 \mathrm{MW}$ & 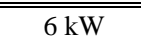 \\
\hline Input dc voltage $\left(V_{d c}\right)$ & $22 \mathrm{kV}$ & $600 \mathrm{~V}$ \\
\hline "Rated current magnitude $\left(I_{o}\right)$ & $1.3 \mathrm{kA}$ & $16.5 \mathrm{~A}$ \\
\hline " Nominal SM capacitor voltage $\left(V_{c}\right)$ & $2.2 \mathrm{kV}$ & $200 \mathrm{~V}$ \\
\hline Fundamental output frequency $\left(f_{o}\right)$ & 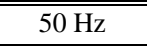 & $\overline{50 \mathrm{~Hz}}$ \\
\hline PWM Carrier frequency $\left(f_{c}\right)$ & $2 \mathrm{kHz}$ & $2 \mathrm{kHz}$ \\
\hline Arm inductance $\left(L_{\text {arm }}\right)$ & $2 \mathrm{mH}$ & $2.4 \mathrm{mH}$ \\
\hline Equivalent SM capacitance $(C)$ & $1 \mathrm{mF}$ & $1.1 \mathrm{mF}$ \\
\hline \multicolumn{3}{|c|}{ DHB parameters } \\
\hline Transformer turns ratio & 1 & 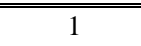 \\
\hline " Auxiliary leakage inductance $(L)$ & 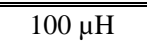 & $70 \mu \mathrm{H}$ \\
\hline Switching frequency $\left(f_{h}\right)$ & $10 \mathrm{kHz}$ & $10 \mathrm{kHz}$ \\
\hline \multicolumn{3}{|c|}{ RL load parameters } \\
\hline Load resistance & $7.88 \times \frac{f_{o}}{50} \Omega$ & $16 \times \frac{f_{o}}{50} \Omega$ \\
\hline Load inductance & $8.25 \mathrm{mH}$ & $26 \mathrm{mH}$ \\
\hline \multicolumn{3}{|c|}{ Motor parameters } \\
\hline Rated output power & $20 \mathrm{MW}$ & $4 \mathrm{kWW}$ \\
\hline Rated line voltage & $13.2 \mathrm{kV}$ & $380 \mathrm{~V}$ \\
\hline Fundamental frequency & $50 \mathrm{~Hz}$ & $50 \mathrm{~Hz}$ \\
\hline Rated speed & $600 \mathrm{rpm}$ & $1440 \mathrm{rpm}$ \\
\hline Number of poles & 10 & 4 \\
\hline
\end{tabular}

Different samples of capacitor voltage for SMs in upper and lower arms are shown balanced around $2.2 \mathrm{kV}$ with $\pm 10 \%$ voltage ripple. The $\mathrm{CM}$ voltage, measured at the terminals of the three-phase load is shown to alternate with near $1 \mathrm{kV}$ amplitude. The supply current is near constant with only switching harmonics, due to the suppression of even harmonics in the arm currents.

Fig. 12 shows the switching waveforms of one DHB module interfacing a pair of upper-arm SMs located in phase $a$ and $b$, within two switching cycles in different time spans. The voltages across the primary and secondary transformer windings are shown, along with the transformer current. The amount of power transfer is in direct proportion to the magnitude of the phase angle difference, while forward or reverse power transfer is designated depending on the polarity of $\delta$.

To highlight the effectiveness of the employed power decoupling scheme, the simulation was performed at low operating frequencies, while the load resistance was varied linearly with the frequency reduction to maintain the load current constant at the rated value. The DHB scheme is initially deactivated, and the MMC system behaves as a conventional MMC, (the results are recorded for one operating cycle). Then, the DHB scheme is activated, and the system performance is monitored for two operating cycles. This is shown in Figs. 13 and 14 for operation at $10 \mathrm{~Hz}$ and $5 \mathrm{~Hz}$, respectively. Common to both cases is that the quality of both line voltages and load currents is enhanced with the activation of the power decoupling scheme. Also, with the fundamental
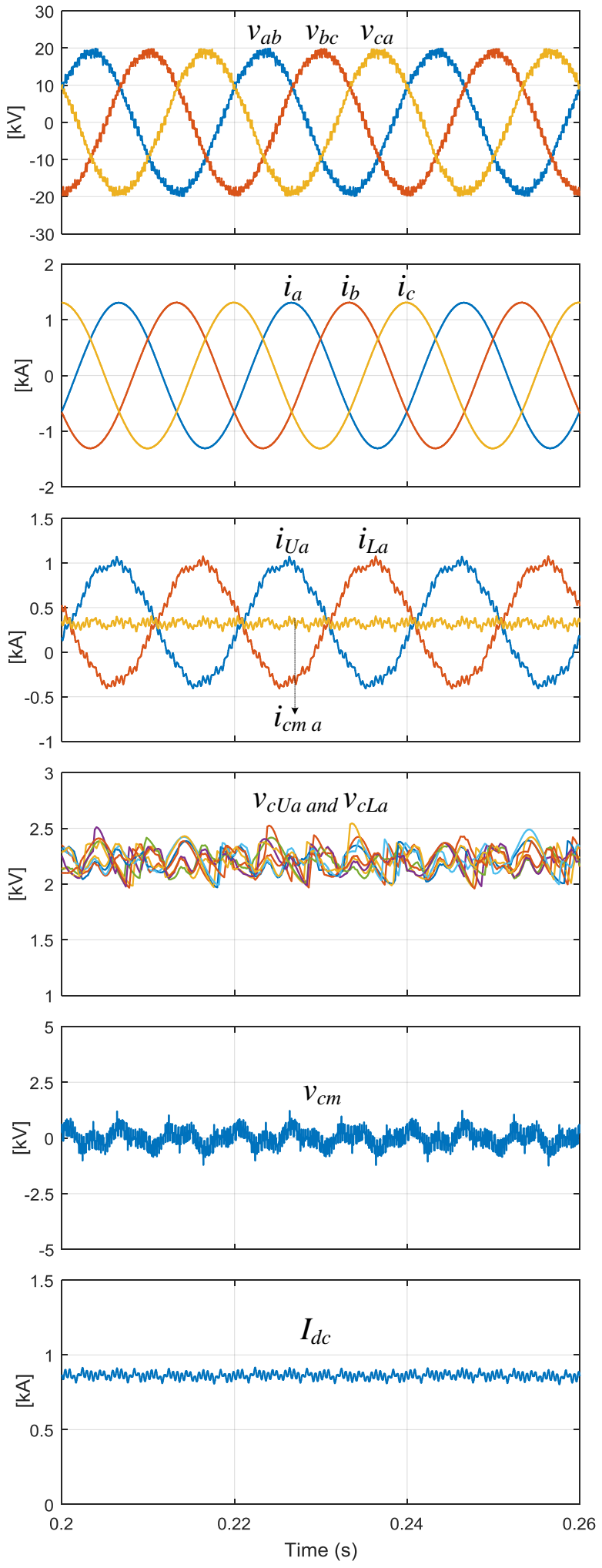

Fig. 11 Simulation results for the MMC configuration at rated parameters.

and second-order arm powers decoupled in the three-phase MMC legs, the arm currents are near even-harmonics-free without a harmonic suppression control algorithm. SM capacitor voltages exhibit significant ripple reduction with the DHB energy balancing scheme, where at $10 \mathrm{~Hz}$, the voltage ripple is reduced from $\pm 52 \%$ to $\pm 5 \%$, and from $\pm 90 \%$ to 

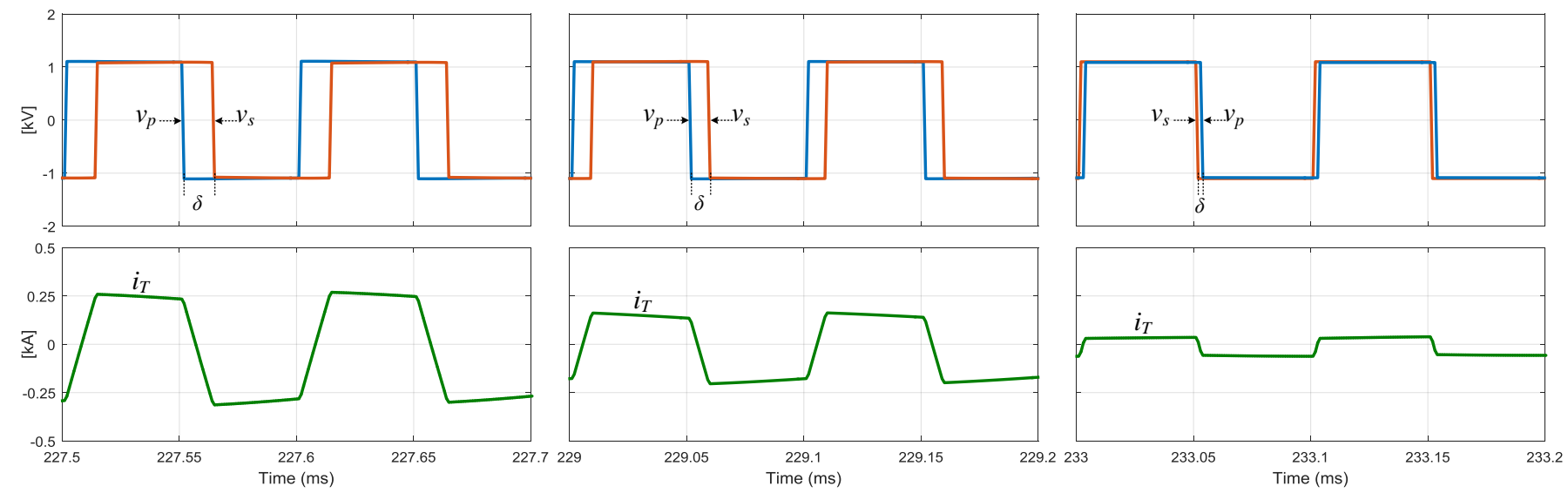

Fig. 12 Simulation results for the switching waveforms of the phase-shift DHB converter at rated parameters.

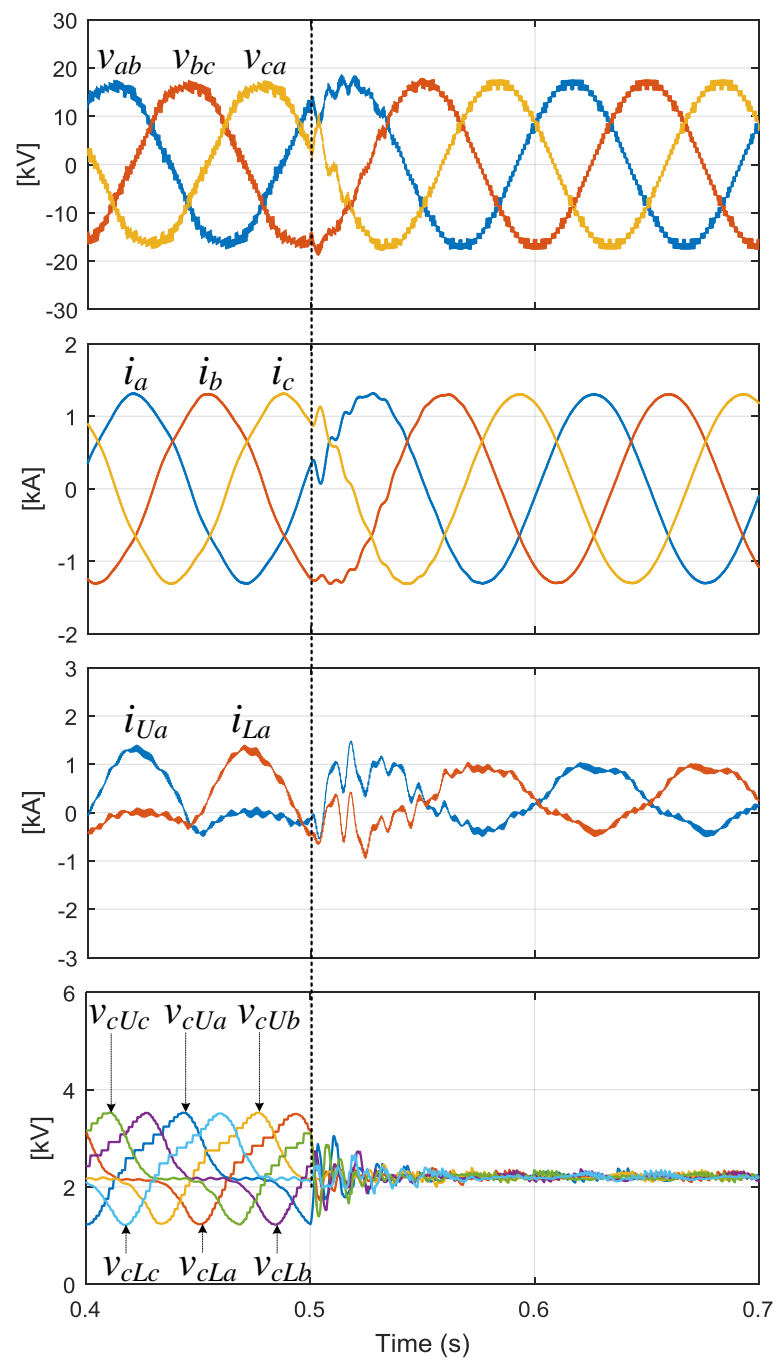

Fig. 13 MMC performance at $10 \mathrm{~Hz}$ before and after DHB power decoupling scheme activation.

$\pm 5 \%$ at $5 \mathrm{~Hz}$. Although operation with such extreme voltage ripple percentages without DHB scheme incorporation is impractical, it gives clear indication of the significant reduction achieved in SM capacitance when the power decoupling scheme is employed.

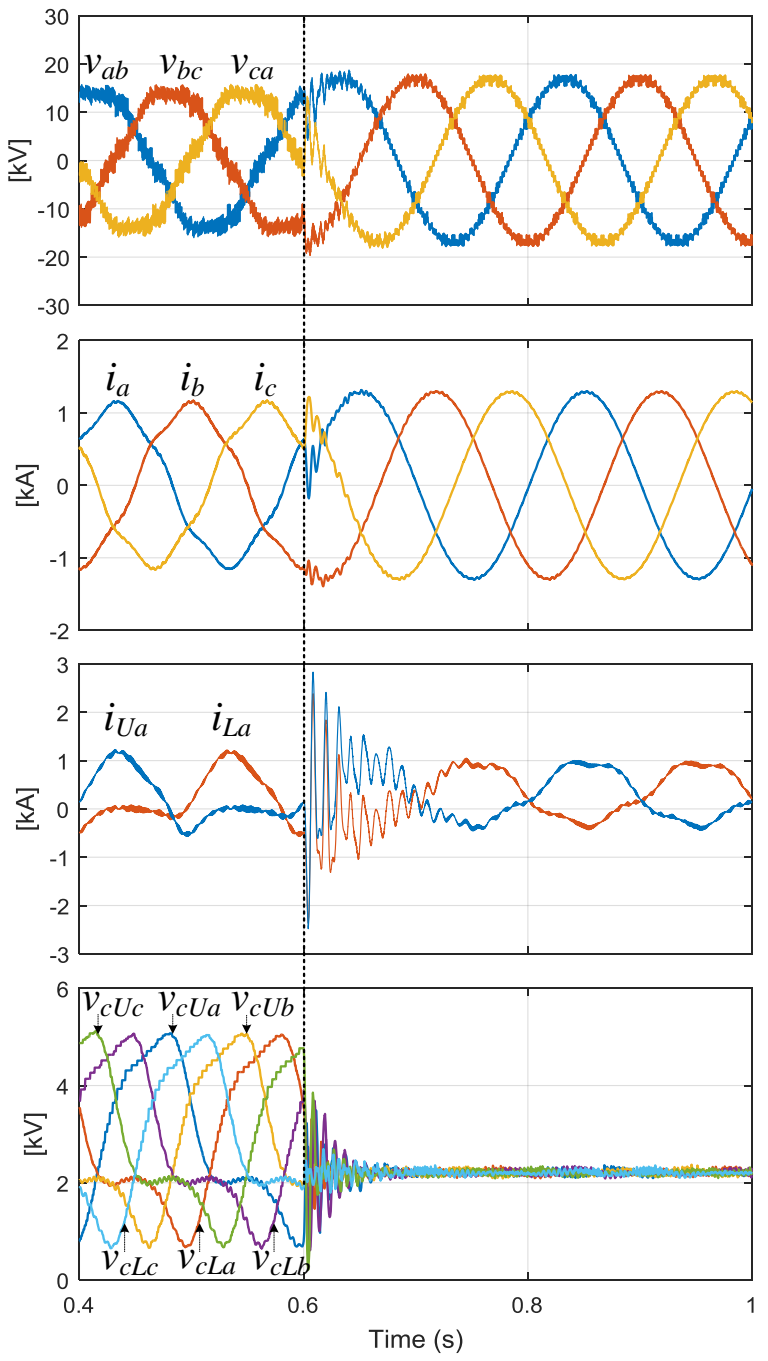

Fig. $14 \mathrm{MMC}$ performance at $5 \mathrm{~Hz}$ before and after DHB power decoupling scheme activation.

Fig. 15 shows the dynamic performance of the proposed MMC-DHB system when driving a three-phase induction machine from standstill to the rated speed, at the rated torque operating condition. The motor currents maintain a highquality sinusoidal profile within the entire speed range. 

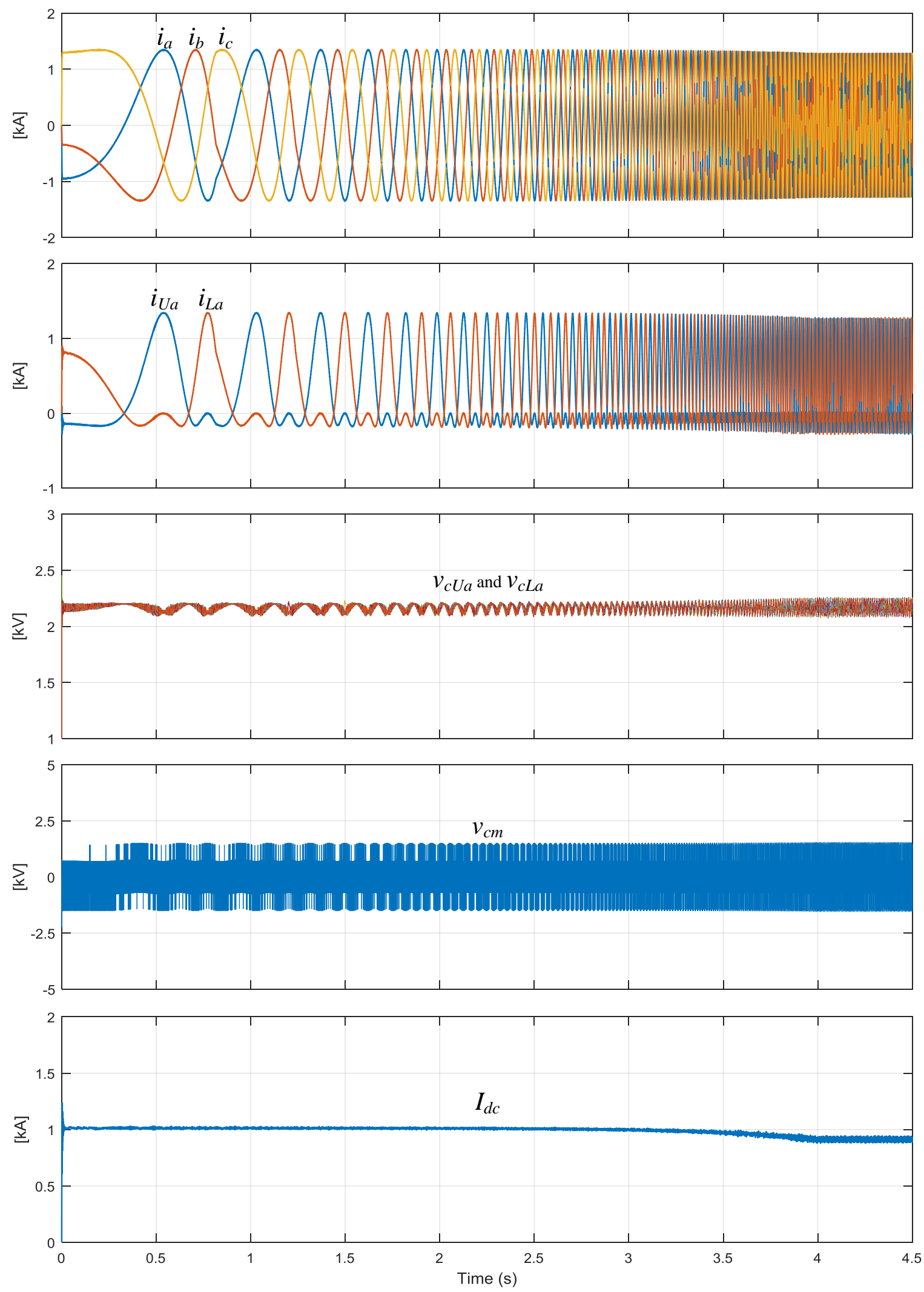

Fig. 15 Dynamic performance of the proposed MMC system driving a 20 MW induction machine from standstill to the rated speed.

The SM capacitor voltage ripple is limited within a very narrow ripple band, even at near zero frequency. The $\mathrm{CM}$ voltage swings with a $1.5 \mathrm{kV}$ amplitude. The supply current is continuous with only switching harmonics, while it is slightly reduced when the motor reaches the steady-state at the rated speed. 


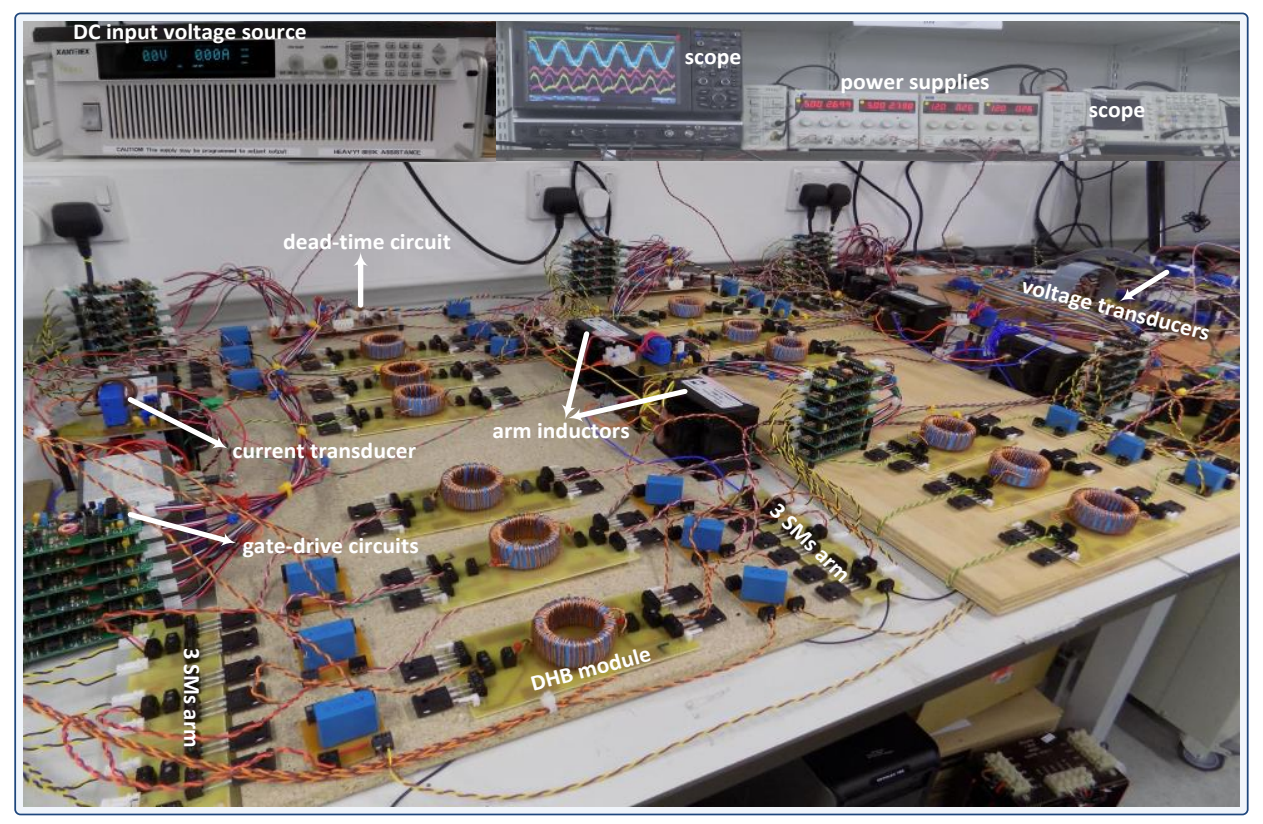

Fig. 16 Hardware view of the experimental prototype.

\section{B. Experimental Assessment}

The experimental downscaled prototype MMC-DHB system comprises a three-phase MMC rated at $6 \mathrm{~kW}, 360 \mathrm{~V}$, with 3 SMs per arm and supplied from a $600 \mathrm{~V}$ dc-link. To reduce the number of employed SM balancers, configuration 2 is adopted for the MMC energy balancing scheme, where each three adjacent-arm SMs are interfaced through two DHB modules, each employing a nanocrystalline core-based HF transformer with a core saturation flux density of $1.2 \mathrm{~T}$ and a unity turns ratio. The control system is implemented in TMS320F28335 DSPs. The experimental setup is shown in Fig. 16. Both steady-state and dynamic performance of the proposed MMCDHB system are examined under different operating scenarios. Steady-state results at different operating frequencies were recorded while the MMC-DHB system is supplying three-phase $R L$ load, where the resistance was varied linearly in proportional to the frequency variation to emulate constant Volt/Hertz operation. Dynamic results were obtained during starting of a three-phase induction machine from standstill to the rated speed. Experimental waveforms are presented in Figs. 17 to 22.

\section{Steady-State Performance}

Fig. 17 shows the fundamental waveforms of the MMC at the rated parameters of Table II, where the MMC is supplying a three-phase $R L$ load at $50 \mathrm{~Hz}$. The phase voltage waveforms are shown with respect to the virtual ground of the dc-link midpoint, so have seven voltage-levels between $\pm 300 \mathrm{~V}$. The $\mathrm{CM}$ voltage is shown as the average of the measured phase voltages with $70 \mathrm{~V}$ amplitude. The three-phase line voltages and corresponding load currents are shown to be balanced. Arm currents of phase $a$ and its CM current are shown, where the arm currents are free of dominant even-harmonics due to $\mathrm{CM}$ current regulation, resulting in a near dc CM current.

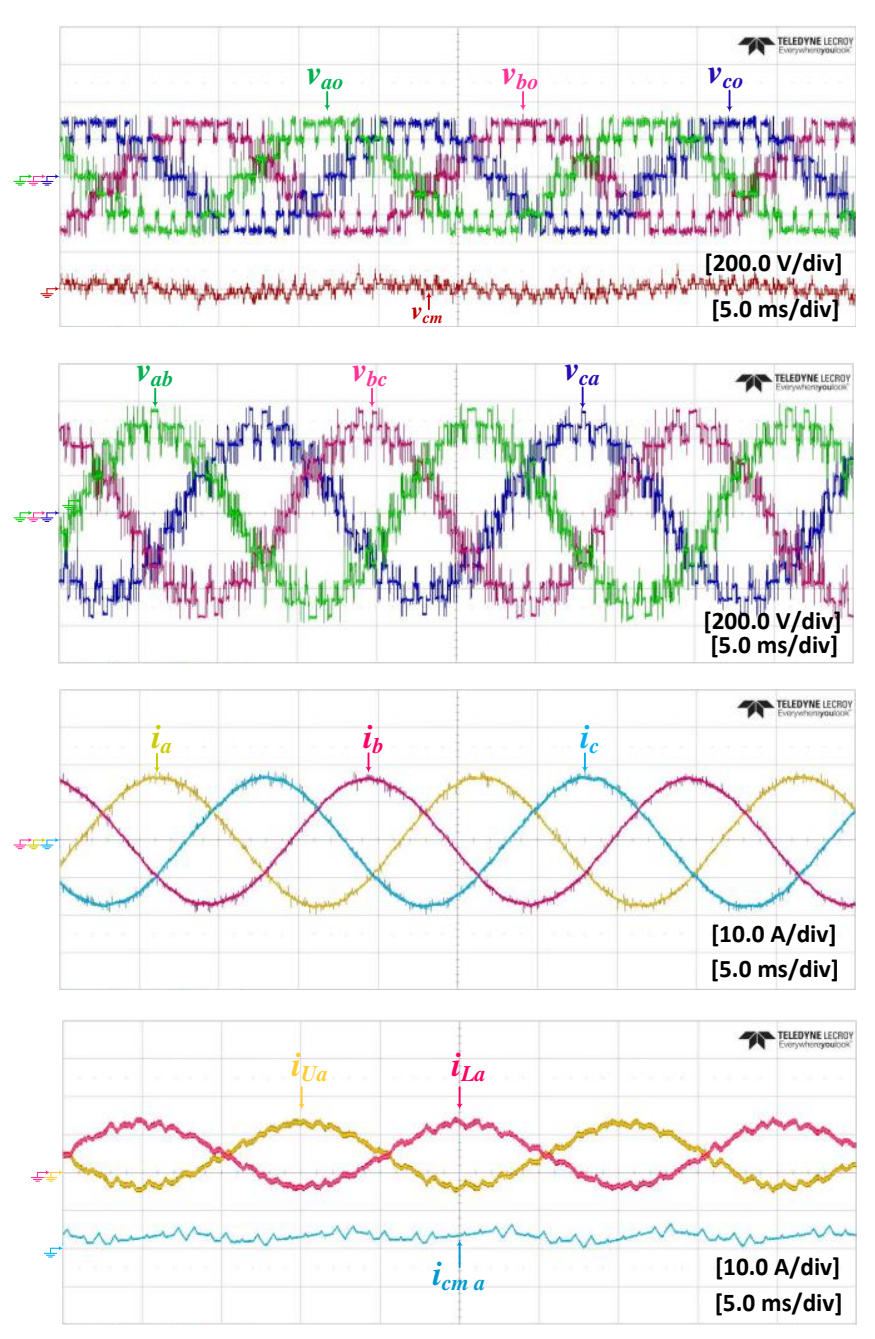

Fig. 17 Experimental waveforms of the MMC system at rated parameters. 


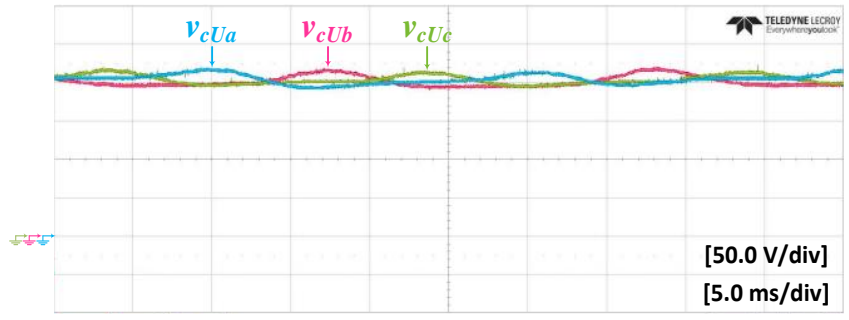

(a)

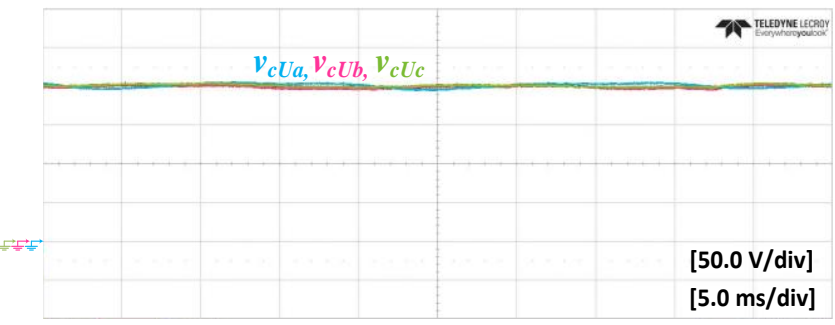

(b)

Fig. 18 Capacitor voltage fluctation at $50 \mathrm{~Hz}$ when the DHB scheme is (a) deactivated and (b) activated.

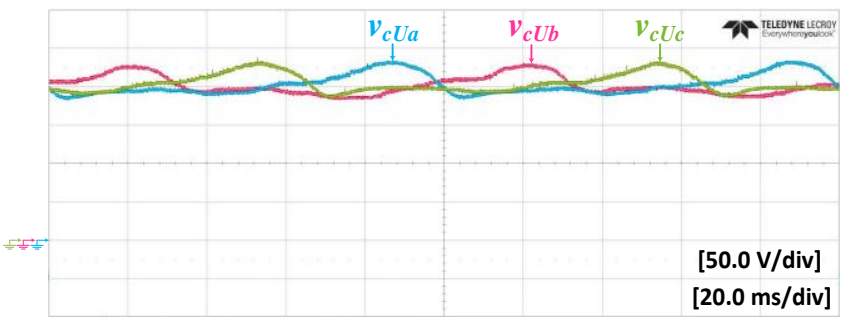

(a)

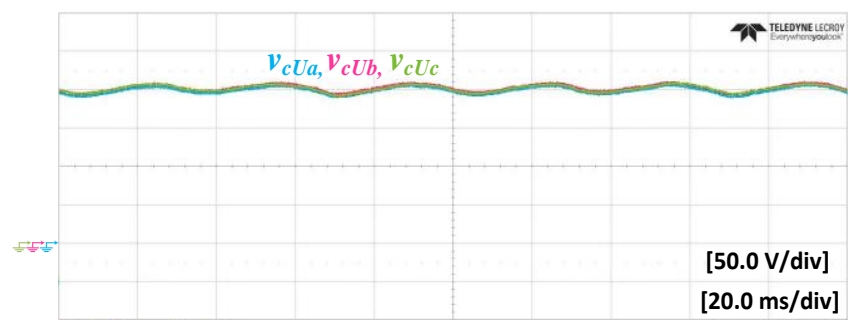

(b)

Fig. 19 Capacitor voltage fluctation at $10 \mathrm{~Hz}$ when the DHB scheme is (a) deactivated and (b) activated.

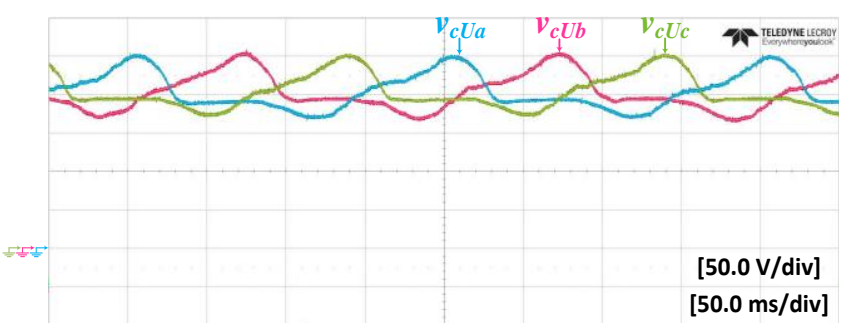

(a)

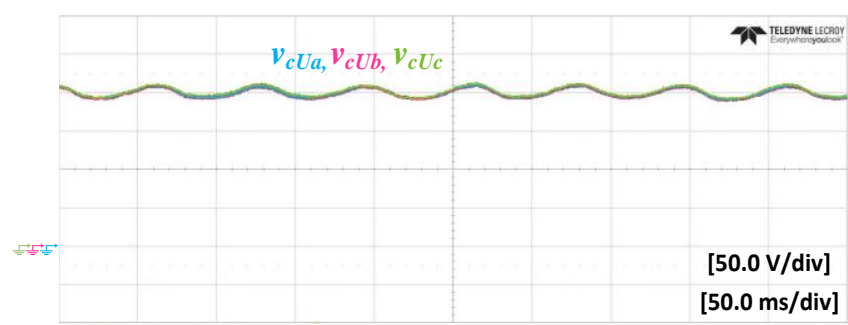

(b)

Fig. 20 Capacitor voltage fluctation at $5 \mathrm{~Hz}$ when the DHB scheme is (a) deactivated and (b) activated.

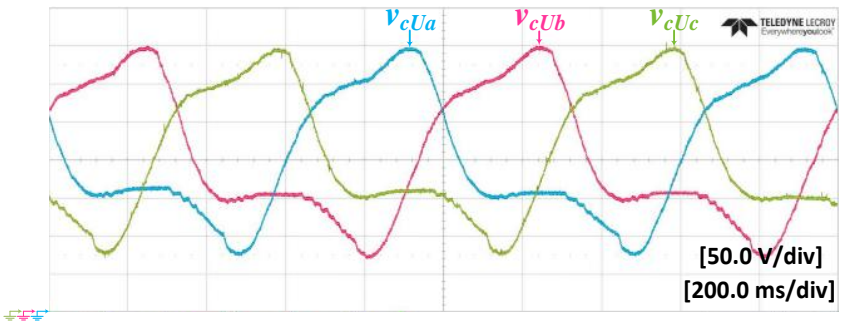

(a)

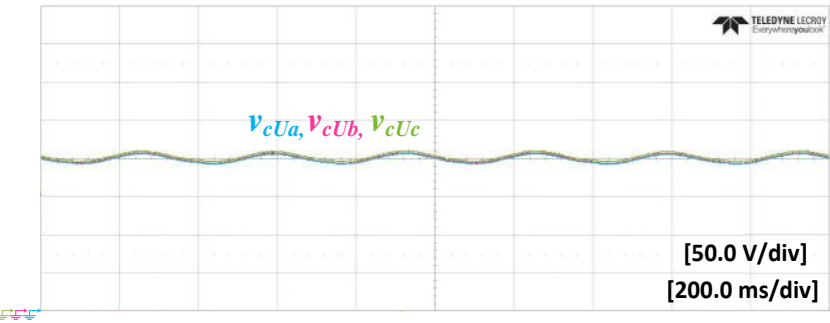

(b)

Fig. 21 Capacitor voltage fluctation at $1 \mathrm{~Hz}$ when the DHB scheme is (a) deactivated and (b) activated.

Figs. 18-21 practically assess the influence of the DHB power decoupling scheme by presenting SM capacitor voltage fluctuation at different operating frequencies when the DHB scheme is deactivated, then operational. Three samples of upper-arm SM capacitor voltages are shown for the three phase-legs. At $50 \mathrm{~Hz}$, Fig. 18 shows that capacitor voltage ripple is reduced from $\pm 5.6 \%$ to $\pm 2.5 \%$ with incorporation of the DHB scheme. A significant reduction in capacitor voltage ripple, with the activation of the energy balancing scheme occurs at reduced operating frequencies, where at $10 \mathrm{~Hz}$ (see Fig. 19) the capacitor voltage ripple decreases from $\pm 11.25 \%$ to $\pm 4.25 \%$, while at $5 \mathrm{~Hz}$ (see Fig. 20) the voltage ripple decreases from $\pm 21 \%$ to $\pm 5.25 \%$. At $1 \mathrm{~Hz}$, Fig. 21 a shows that the capacitor voltage ripple using a conventional MMC is extreme, $\pm 67 \%$, possibly threatening the safe operation of the
MMC components and results in a loss of system controllability. The DHB scheme efficiently restores the MMC balance as shown in Fig. 21b, where the capacitor voltage ripple is $\pm 6 \%$. Although the capacitor voltage ripple with the DHB scheme is in operation slightly increases with frequency reduction, the ripple is maintained within tolerated limits at all operating frequencies.

Fig. 22 shows the waveforms of one DHB converter at rated parameters. The square wave voltages across the primary and secondary sides of the HF transformer are shown along with the transformer current at a time instant when the primary-side $\mathrm{HB}$ is leading the secondary-side HB. The switching waveforms across the IGBTs are shown to illustrate the ZVS. Both gate-to-emitter $\left(v_{G E}\right)$ and collector-to-emitter $\left(v_{C E}\right)$ voltages are shown at the DHB converter primary side. 


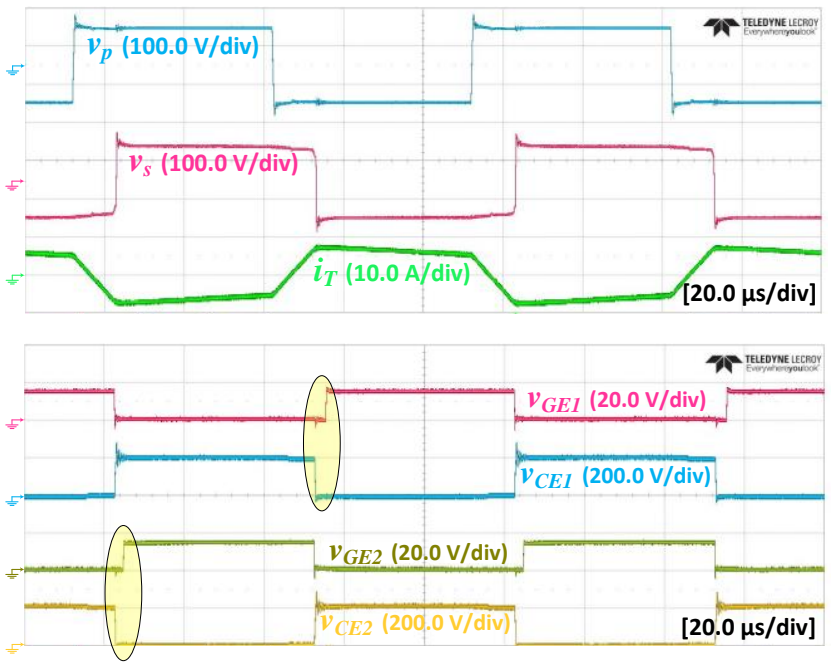

Fig. 22 DHB converter switching waveforms at rated parameters.

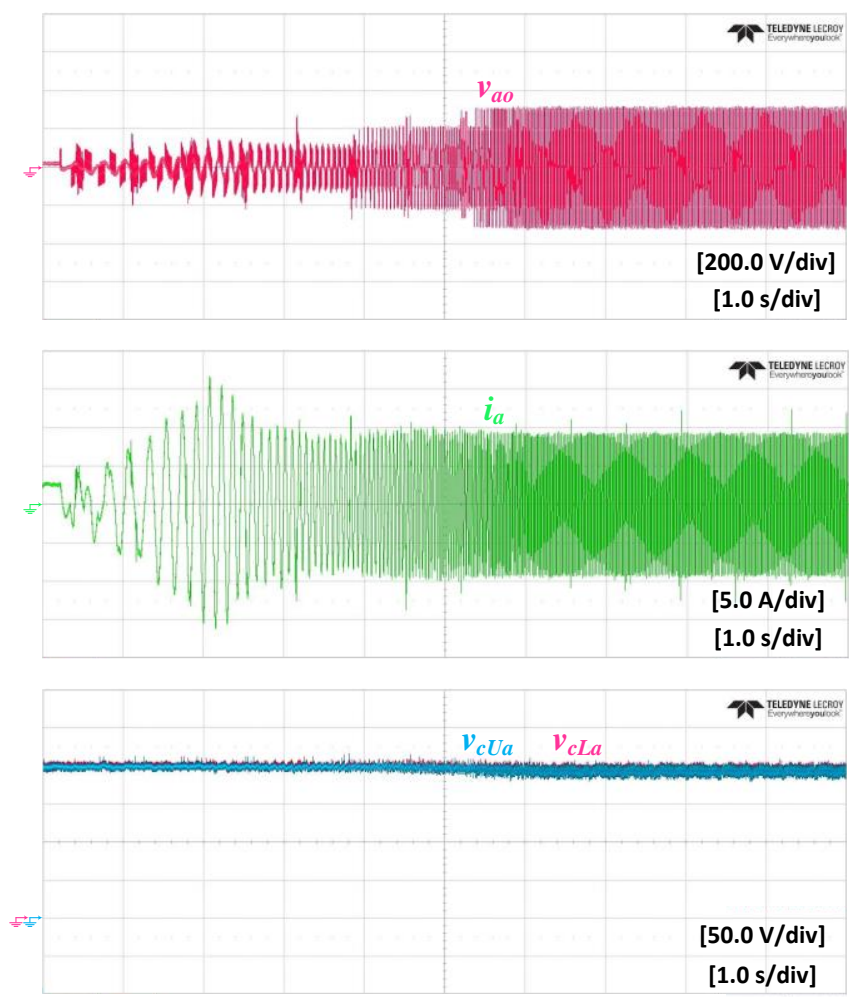

Fig. 23 Dynamic performance of the proposed drive system when starting an induction motor from standstill to the rated speed at rated torque.

\section{Dynamic Performance}

The dynamic performance of the proposed MMC-DHB system is experimentally examined as a motor acceleration process from standstill to the rated speed, at the full load torque throughout, as shown in Fig. 23. The phase voltage and motor current are recorded from phase $a$, where the MMC system, with the aid of the DHB power decoupling scheme, is able to generate a near dc voltage as briefly as required by the motor at zero speed with an acceptable SM capacitor voltage ripple profile. Sample upper and lower capacitor voltages are shown to have a near constant ripple profile within the entire speed range, where the ripple voltage is limited within $\pm 5 \%$.

\section{CONCLUSION}

In this paper, a decoupling approach for MMC capacitor voltage ripple compensation has been proposed for threephase MMC-fed variable speed drives. The approach employs magnetic chain links equipped with DHBs to interface adjacent-arm SMs. Based on their symmetrical alternation, the ripple powers of the MMC phase legs are decoupled altogether within each chain of three magnetically-coupled SMs, where the ripple power is transferred from one SM to another to compensate for the energy imbalance. This results in a significant enhancement in the SM capacitor voltage profile, where the voltage ripple is brought down to a very narrow band, which inherently reduces the even-order harmonics in the CM current. A significant reduction in the SM capacitance is possible since the voltage ripple can be considered near constant, independent of the operating frequency. The proposed MMC-DHB configuration is efficiently able to drive multi-megawatt machines continuously at low operating speeds, from standstill condition, at the rated torque. Two MMC-DHB configuration variations have been presented, each with a different number of DHB balancing units, both able to entirely decouple the fundamental and the secondorder ripple power of the MMC arms. One drawback of the proposed MMC-DHB approach is that the system reliability is affected, compared to a traditional MMC system, since the failure of any MMC SM implies deactivating the other SMs and DHB balancers at the same level within the associated chain. The mathematical analysis of the proposed approach has been presented, and the MMC-DHB drive system performance has been verified through simulation and experimentation, at different operating scenarios.

\section{REFERENCES}

[1] S. Debnath, J. Qin, B. Bahrani, M. Saeedifard, and P. Barbosa, "Operation, control, and applications of the modular multilevel converter: A review," IEEE Trans. Power Electron., vol. 30, no. 1, pp. 37-53, Jan. 2015.

[2] A. Bendre, G. Venkataramanan, D. Rosene, and V. Srinivasan, "Modeling and design of a neutral-point voltage regulator for a threelevel diode clamped inverter using multiple-carrier modulation," IEEE Trans. Ind. Electron., vol. 53, no. 3, pp. 718-726, Jun. 2006.

[3] I.-D. Kim, E.-C. Nho, H.-G. Kim, and J. S. Ko, "A generalized Undeland snubber for flying capacitor multilevel inverter and converter," IEEE Trans. Ind. Electron., vol. 51, no. 6, pp. 1290-1296, Dec. 2004.

[4] S. Sirisukprasert, J. S. Lai, and T. H. Liu, "Novel cascaded multilevel converter drive system with minimum number of separated DC sources," in Proc. IEEE PESC, Vancouver, BC, Canada, Jun. 17-22, 2001, pp. 1346-1350.

[5] A. Lesnicar and R. Marquardt, "An innovative modular multilevel converter topology suitable for a wide power range," in Proc. IEEE Power Tech. Conf., Bologna, Italy, Jun. 23-26, 2003, vol. 3.

[6] A. Korn, M. Winkelnkemper, and P. Steimer, "Low output frequency operation of the modular multi-level converter", 2010 IEEE Energy Conversion Congress and Exposition, Atlanta, GA, 2010, pp. 39933997.

[7] S. Debnath, J. Qin and M. Saeedifard, "Control and Stability Analysis of Modular Multilevel Converter under Low-Frequency Operation," IEEE Trans. Ind. Electron, vol. 62, no. 9, pp. 5329-5339, Sept. 2015.

[8] J. Kolb, F. Kammerer, M. Gommeringer, and M. Braun, "Cascaded control system of the modular multilevel converter for feeding variablespeed drives," IEEE Trans. Power Electron., vol. 30, no. 1, pp. 349-357, Jan. 2015.

[9] A. Antonopoulos, L. Angquist, S. Norrga, K. Ilves, L. Harnefors, H.-P. Nee, "Modular multilevel converter ac motor drives with constant torque 
from zero to nominal speed," IEEE Trans. Ind. Appl., vol. 50, no. 3, pp. 1982-1993, May/Jun. 2014.

[10] K. Wang, Y. Li, Z. Zheng, and L. Xu, "Voltage balancing and fluctuation-suppression method of floating capacitors in a new modular multilevel converter," IEEE Trans. Ind. Electron., vol. 60, no. 5, pp. 1943-1954, May 2013.

[11] B. Li, S. Zhou, D. Xu, R. Yang, D. Xu, C. Buccella, and C. Cecati, “An improved circulating current injection method for modular multilevel converters in variable-speed drives" IEEE Trans. Ind. Electron., vol. 63, no. 11, pp. 7215-7225, Nov. 2016.

[12] A. Antonopoulos, L. Ängquist, L. Harnefors and H. P. Nee, "Optimal Selection of the Average Capacitor Voltage for Variable-Speed Drives with Modular Multilevel Converters," IEEE Trans. Power Electron., vol. 30, no. 1, pp. 227-234, Jan. 2015.

[13] B. Tai; C. Gao; X. Liu; Z. Chen, "A Novel Flexible Capacitor Voltage Control Strategy for Variable-Speed Drives with Modular Multilevel Converters," IEEE Trans. Power Electron., vol. 32, no. 1, pp. 128-141, Jan. 2017.

[14] Yang; B. Li; G. Wang; C. Cecati; S. Zhou; D. G. Xu; W. Yu, "Asymmetric mode control of MMC to suppress capacitor voltage ripples in low frequency low voltage condition," IEEE Trans. Power Electron., vol. 32, no. 6, pp. 4219-4230, June 2017.

[15] A. Mertens and J. Kucka, "Quasi Two-Level PWM Operation of an MMC Phase Leg with Reduced Module Capacitance," IEEE Trans. on Power Electron., vol. 31, no. 10, pp. 6765-6769, Oct. 2016.

[16] S. Du, B. Wu, K. Tian, N. R. Zargari and Z. Cheng, "An Active CrossConnected Modular Multilevel Converter (AC-MMC) for a MediumVoltage Motor Drive," IEEE Trans. Ind. Electron, vol. 63, no. 8, pp. 4707-4717, Aug. 2016

[17] S. Du; B. Wu; N. Zargari; Z. Cheng, "A Flying-Capacitor Modular Multilevel Converter (FC-MMC) for Medium-Voltage Motor Drive," IEEE Trans. Power Electron., vol. 32, no. 3, pp. 2081-2089, March 2017.

[18] Z. Kong; X. Huang; Z. Wang; J. Xiong; K. Zhang, "Active Power Decoupling for Submodules of Modular Multilevel Converter," IEEE Trans. Power Electron, vol. 33, no. 1, pp. 125-136, Jan. 2018

[19] L. He, K. Zhang, J. Xiong, S. Fan, and Y. Xue, "Low-frequency ripple suppression for medium-voltage drives using modular multilevel converter with full-bridge submodules," IEEE J. Emerg. Sel. Topics Power Electron., vol. 4, no. 2, pp. 657-667, June 2016.

[20] M. S. Diab, A. M. Massoud, S. Ahmed and B. W. Williams, "A Dual Modular Multilevel Converter With High-Frequency Magnetic Links Between Submodules for MV Open-End Stator Winding Machine Drives," IEEE Trans. Power Electron., vol. 33, no. 6, pp. 5142-5159, June 2018.

[21] L. He, K. Zhang, J. Xiong, S. Fan, X. Chen and Y. Xue, "New modular multilevel converter with power channels between upper- and lower arms suitable for MV drives," 2015 IEEE Applied Power Electronics Conference and Exposition (APEC), Charlotte, NC, 2015, pp. 799-805.

[22] A. Zafeiropoulos, A. Antonopoulos and J. R. Svensson, "An MMCbased topology using DHB power channels for load balancing in $50 \mathrm{~Hz}$ railway applications," 2017 IEEE Energy Conversion Congress and Exposition (ECCE), Cincinnati, OH, 2017, pp. 83-90.

[23] Z. Li, P. Wang, Z. Chu, H. Zhu, Y. Luo, and Y. Li, "An inner current suppressing method for modular multilevel converters," IEEE Trans. Power Electron., Vol. 28, No. 11, pp. 4873-4879, Nov. 2013.

[24] M. S. Diab, B. W. Williams, D. Holliday, A. M. Massoud and S. Ahmed, "A modular multilevel converter with isolated energy-balancing modules for MV drives incorporating symmetrical six-phase machines," 2017 IEEE Energy Conversion Congress and Exposition (ECCE), Cincinnati, OH, 2017, pp. 2715-2722.

[25] M. H. Kheraluwala, R.W. Gascoigne, D.M. Divan and E. D. Baumann, "Performance characterization of a high-power dual active bridge dc-todc converter," IEEE Trans. Ind. Applications, vol. 28, no. 6, pp. 12941301, Nov/Dec. 1992.

[26] H. Li, F. Z. Peng and J. S. Lawler, "A natural ZVS medium-power bidirectional DC-DC converter with minimum number of devices," IEEE Trans. Ind. Appl., vol. 39, no. 2, pp. 525-535, Mar/Apr 2003.

[27] F. Z. Peng, Hui Li, Gui-Jia Su and J. S. Lawler, "A new ZVS bidirectional DC-DC converter for fuel cell and battery application," IEEE Trans. Power Electron., vol. 19, no. 1, pp. 54-65, Jan. 2004.

[28] A. Antonopoulos, L. Angquist, and H.-P. Nee, "On dynamics and voltage control of the modular multilevel converter," 2009 13th
European Conference on Power Electronics and Applications, Barcelona, 2009, pp. 1-10.

[29] M. Leibl, G. Ortiz and J. W. Kolar, "Design and Experimental Analysis of a Medium-Frequency Transformer for Solid-State Transformer Applications," IEEE J. Emerg. Sel. Topics Power Electron., vol. 5, no. 1, pp. 110-123, March 2017.

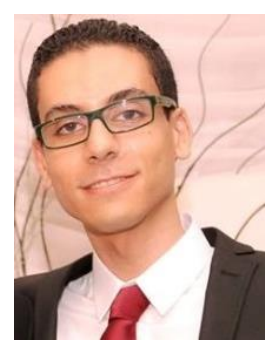

Mohamed S. Diab (S'09) received the B.Sc. (First Class Hons.) and M.Sc. degrees in electrical engineering from Alexandria University, Alexandria, Egypt, in 2012, and 2015, respectively. $\mathrm{He}$ is currently working toward the Ph.D. degree in electrical engineering at the University of Strathclyde, Glasgow, U.K. He was in the Department of Electrical Engineering, Alexandria University, where he was appointed as a Demonstrator in 2012 and as an Assistant Lecturer in 2015. He was with Spiretronic LLC, Houston, TX, USA, as a Research Engineer, from 2013 to 2015. His main research interests include medium-voltage applications, high-power electronic converters, renewable energy conversion systems, grid integration of distributed generators, and electric drives.

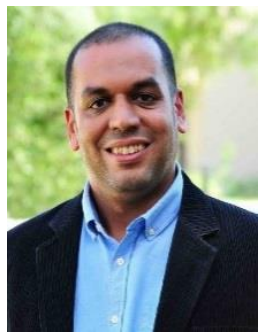

Ahmed M. Massoud (SM'11) received the B.Sc. (First Class Hons.) and M.Sc. degrees in electrical engineering from Alexandria University, Alexandria, Egypt, in 1997 and 2000, respectively, and the Ph.D. degree in electrical engineering from Heriot-Watt University, Edinburgh, U.K., in 2004. $\mathrm{He}$ is currently an Associate Professor at the Department of Electrical Engineering, College of Engineering, Qatar University. His research interests include power electronics, energy conversion, renewable energy and power quality. He holds five U.S. patents. He published more than 100 journal papers in the fields of power electronics, energy conversion, and power quality.

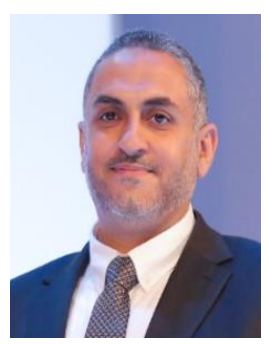

Shehab Ahmed (SM'12) received the B.Sc. degree from Alexandria University, Alexandria, Egypt, in 1999, and the M.Sc. and Ph.D. degrees from the Department of Electrical and Computer Engineering, Texas A\&M University, College Station, TX, USA, in 2000 and 2007, respectively, all in electrical engineering. He was with Schlumberger Technology Corporation, Houston, TX, USA, from 2001 to 2007, where he was involved in downhole mechatronic systems. He is currently an Associate Professor with Texas A\&M University at Qatar, Doha, Qatar. His research interests include mechatronics, solid-state power conversion, electric machines, and drives.

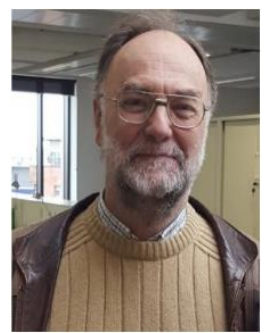

Barry W. Williams received the M.Eng.Sc. degree from the University of Adelaide, Adelaide, S.A., Australia, in 1978, and the Ph.D. degree from Cambridge University, Cambridge, U.K., in 1980, both in electrical engineering. After seven years as a Lecturer with Imperial College, University of London, London, U.K., he was appointed to a Chair of the Electrical Engineering, Heriot-Watt University, Edinburgh, U.K., in 1986. He is currently a Professor with the University of Strathclyde, Glasgow, U.K. His teaching covers power electronics (in which he has a free internet text) and drive systems. His research interests include power semiconductor modeling and protection, converter topologies, soft-switching techniques, and application of ASICs and microprocessors to industrial electronics. 